\title{
PHASE AND AMPLITUDE COMPUTATIONS IN THE MIDBRAIN OF AN ELECTRIC FISH: INTRACELLULAR STUDIES OF NEURONS PARTICIPATING IN THE JAMMING AVOIDANCE RESPONSE OF EIGENMANNIA
}

\author{
WALTER HEILIGENBERG ${ }^{2}$ AND GARY ROSE \\ Neurobiology Unit, Scripps Institution of Oceanography, University of California at San Diego, La Jolla, California 92093
}

Received May 22, 1984; Revised July 19, 1984; Accepted July 25, 1984

\begin{abstract}
Electric fish monitor modulations in sensory feedback from their own electric organ discharges (EODs) to locate moving objects and to detect interfering EODs of their neighbors. The gymnotoid genus Eigenmannia minimizes detrimental effects of jamming by EODs of its neighbors by shifting its own EOD frequency away from a neighbor's EOD frequency that is too close to its own. Since the animal lowers its own frequency if its neighbor's frequency is higher and raises its frequency if its neighbor's frequency is lower, this jamming avoidance response (JAR) requires that the animal determine the sign of the difference frequency, $D f$, between the interfering EODs. Eigenmannia obtains this information by evaluating modulations in the amplitude and phase which its nearly sinusoidal EOD signal experiences due to the interference with the neighbor's EODs. The necessary logical operations are executed in the dorsal torus semicircularis, an analogue of the inferior colliculus of higher vertebrates, and are similar to operations underlying directional hearing.

By intracellular labeling of physiologically identified cells we have identified the anatomy and functional characteristics of neurons involved in the processing of amplitude and phase information. The JAR is controlled by hierarchical and parallel processing of information in several laminae of somatotopically ordered neurons. Phase differences between signals received by electroreceptors in different parts of the body surface are computed in lamina 6 . Information about differential phase is then relayed to multipolar cells in the deeper laminae $8, \mathrm{~b}$ and $\mathrm{c}$, which also receive information about modulations in local signal amplitude. These cells are excited by a rise or fall of amplitude as well as by a lead or lag of phase. According to their responses to either of these two variables, these neurons can be divided into four classes. These classes encode all information necessary for the control of the JAR and project to the optic tectum.

Dynamic properties and sensory specificities of the JAR are not found in individual, properly tuned neurons but rather emerge statistically from the joint effects of a large population of imprecisely tuned neurons. This system is characterized by a distributed pattern of organization and by the absence of a small number of key neurons whose malfunction would jeopardize the behavioral response. The complexity of this neural machinery appears unnecessary for the logically simple task of the JAR, and it suggests that this behavior was acquired later in evolution by being derived from more general motor responses to moving objects. The emergence of the JAR thus required modifications of and additions to a neural machinery which already was adapted for other functions.
\end{abstract}

The identification of neural operations involved in the evaluation of sensory stimulus patterns and the control of adaptive behavioral responses represents a major goal in neurobiology. This pursuit should always start with experimental manipulation of the stimulus pattern and behavioral tests on the intact animal in order to determine the relevance of stimulus variables

\footnotetext{
${ }^{1}$ We thank Catherine Carr, John Dye, and Theodore H. Bullock for their excellent advice on anatomical and physiological issues of this study. Grace Kennedy and Margaret Leong processed histological material with great diligence and patience. This study was supported by National Science Foundation Grant BNS82-05454 to W. H. and National Institutes of Health Postdoctoral Fellowship NS 07261 to G. R.

${ }^{2}$ To whom correspondence should be addressed.
}

as well as the nature of their evaluation by the animal. This approach enables us to make rigorous statements about logical operations that must be executed in the animal's nervous system. It also guides our search for the specific neuronal implementations of such operations in that it provides a catalogue of precise tests which we must apply to neurons believed to take part in the control of this behavior. Starting out at the receptor level and gradually advancing to higher-order neurons, we can then trace the succession of neuronal operations which ultimately lead to the execution of the behavioral response.

The jamming avoidance response (JAR; Bullock et al., 1972) in the weakly electric fish, Eigenmannia, represents an extremely promising system in which to pursue this goal since it is a behavior which persists in neurophysiological preparations and can thus be monitored continually while individual neurons 
are being recorded from and labeled intracellularly. Eigenmannia produces nearly sinusoidal electric organ discharges (EODs) at a very stable fundamental frequency (Bullock et al., 1975). Each discharge cycle is triggered by a command from a medullary pacemaker nucleus (Bennett, 1971), and the firing frequency of this nucleus is thus identical to the fundamental or repetition frequency of the EOD. This frequency varies among individuals over a range from 250 to $700 \mathrm{~Hz}$. The JAR is a gradual shift of the animal's EOD frequency away from a similar frequency of a near neighbor. Since the animal lowers its own frequency if its neighbor's frequency is higher and raises its frequency if its neighbor's frequency is lower, it must be able to discriminate the sign of the frequency difference, $D f$, between the two interfering signals. Numerous behavioral experiments have demonstrated that the animal is able to determine the sign of this frequency difference by evaluating modulations in instantaneous phase and amplitude in the nearly sinusoidal signal which results from the interference of the two EODs in all parts of its body surface. All that is needed is that the animal be able to detect local rises and falls in the amplitude of the nearly sinusoidal stimulus and to determine whether the phase (i.e., the timing of zero-crossings) of this stimulus leads or lags that of the stimulus in some other part of its body surface (for a review of extensive behavioral studies, see Heiligenberg, 1980, 1983). The JAR thus requires detection of modulations in amplitude and modulations in differential phase in signals perceived in different parts of the animal's sensory surface and, thus, shares certain organizational principles with auditory phenomena, such as directional hearing.

Neurophysiological and anatomical studies on the electrosensory lateral line lobe (ELLL) of the hindbrain and the torus semicircularis of the midbrain have shown that information about local modulations of stimulus amplitude, conveyed by $\mathbf{P}$ type receptors (Scheich et al., 1973), is coded by pyramidal cells in the somatotopically ordered ELLL and relayed to laminae $3,5,7$, and 8 of the somatotopically ordered torus. Information about local modulations of stimulus phase, conveyed by T-type receptors, is coded by the spherical cells of the ELLL and relayed to lamina 6 of the torus (Carr et al. 1981, 1983; see review by Heiligenberg and Bastian, 1984). Extracellular recordings in the torus have demonstrated the existence of cells which respond differentially to the sign of the frequency difference, $D f$, between interfering signals, and it was concluded that this required evaluation of differences in the phase of signals arriving from separate areas of the body surface (Bastian and Heiligenberg 1980a, b; Partridge et al., 1981). These cell types have now been identified by intracellular recording and labeling. In addition, we have applied stimulus regimens which have allowed us to provide modulations in stimulus phase without concurrent modulations in amplitude and vice versa in order to test a neuron's response to modulations of either modality alone as well as to their normal concurrence.

We have encountered a variety of cell types which probably participate in the control of the JAR but, in addition, may also play a role in the analysis of electric images which originate from moving objects. The computation of phase and amplitude information does not appear to be carried out in the simplest manner required for the control of the JAR, and the nature of the overly complex design of the neuronal organization of the torus raises thoughts about the evolutionary origin of this behavior as well as about statistical rules governing the development of this structure.

\section{Materials and Methods}

Our experimental procedures followed those used in earlier intracellular studies (Heiligenberg and Dye, 1982). Animals of the genus Eigenmannia, ranging in length from 8 to $18 \mathrm{~cm}$, were immobilized by intramuscular injection of 1 to $2 \%$ Flaxedil, gently suspended under the water surface, and respirated by a constant flow of water through the mouth. A small Plexiglas holder was glued to a parietal bone to mechanically stabilize the head for intracellular recording. A small hole, less than $1 \mathrm{~mm}$ in diameter, was drilled through the thin bone covering the brain area to be studied, and a silver ground wire was placed at the edge of this hole. Electrodes were pulled on a Brown Flaming micropipette puller and their tips were filled with either a 10 to $20 \%$ solution of Lucifer Yellow in $0.1 \mathrm{M} \mathrm{LiCl}$ or a 2 to $10 \%$ solution of horseradish peroxidase (HRP) in $0.5 \mathrm{M} \mathrm{KCl}$ and $0.1 \mathrm{M}$ Tris buffer (pH 7.4). The electrodes had an initial resistance of 300 to 700 megohms and were commonly bevelled in a stream of a suspension of 0.05 Micron Gamma Alumina (Buehler Microlpolish) to lower their resistance to 200 to 400 megohms.

Neurons were penetrated by applying a brief positive current pulse in the case of Lucifer Yellow electrodes and by a brief negative current pulse in the case of IIRP electrodes. Responses of a neuron to various stimulus regimens were tape recorded, and the neuron was then filled by iontophoretic injection with a DC-offset sine wave current, at a frequency of $3 \mathrm{~Hz}$ and peak-to-peak amplitude of $2 \mathrm{nA}$ of appropriate polarity, over a period of 1 to $10 \mathrm{~min}$.

In many instances we were able to record small extracellular spikes before penetrating the cell. We then compared the stimulus response patterns obtained before and after penetration in order to test whether penetration of the membrane altered the response characteristics of the neuron. With the exception of rare cases where cells began to fire erratically or ceased to fire altogether, their response characteristics did not change as a consequence of penetration. Very commonly neurons increased their resting potential and spike size in the course of recording as, apparently, their membrane sealed and the cell recovered after penetration.

Since neurons once filled with Lucifer Yellow begin to disintegrate within a few hours, we perfused animals within $1 \mathrm{hr}$ after injection of a particularly important type of neuron.

Because the animal's EODs are silenced by injection of curare-like drugs, its electroreceptors can be driven exclusively by artificial electrical stimuli. As in previous experiments (Heiligenberg and Bastian, 1980), we substituted a sine wave stimulus applied between an internal and an external electrode for the animal's EOD so that current penetrated the skin fairly perpendicularly and in the same direction at every moment, much as in the case of the natural EOD. The amplitude of the stimulus voltage was adjusted to that of the animal's own EOD prior to curarization. Although the animal's natural EOD contains higher harmonics and is thus not purely sinusoidal, its replacement by a pure sine wave has no detectable consequences upon its JAR performance (Heiligenberg et al., 1978). Details of these stimulus regimens will be given in connection with the experimental results.

\section{Results}

Theoretical considerations and experimental designs. As the animal's EOD or its sinusoidal substitute, $S_{1}$ interferes with a neighbor's EOD or its substitute, $\mathrm{S}_{2}$, a nearly sinusoidal signal $\left(S_{1}+S_{2}\right)$ is obtained whose instantaneous amplitude (or beat envelope) is modulated at the difference frequency, $D f$, of the two interfering signals. Due to differences in the geometry of the $S_{1}$ field and the $S_{2}$ field with respect to the animal's body shape, the angle between the local current vectors of $S_{1}$ and $S_{2}$ varies as a function of space, and, consequently, the local intensity ratio between $S_{2}$ and $S_{1}$ experienced by electroreceptors varies over the animal's body surface. Assume that this ratio is higher in area A than in area B; i.e., that the animal's signal is more heavily contaminated by the foreign signal, $\mathrm{S}_{2}$, in area $A$ than in area $B$. This will then result in a greater modulation of the instantaneous amplitude in $\mathrm{A}$ than in $\mathrm{B}$. If the local instantaneous amplitude of the stimulus, $S_{1}+S_{2}$, within area $A$ is plotted on an ordinate, while the instantaneous difference in stimulus phase, or the timing of zero-crossings, between area $A$ and area $B$ is plotted on the abscissa, data points are obtained which form a nearly circular graph in the manner of a Lissajous figure as time progresses. This graph rotates counterclockwise or clockwise if the frequency of $S_{2}$ is higher or lower, respectively, than the frequency of $S_{1}$, and the rate of rotation is the absolute value of the frequency difference, 


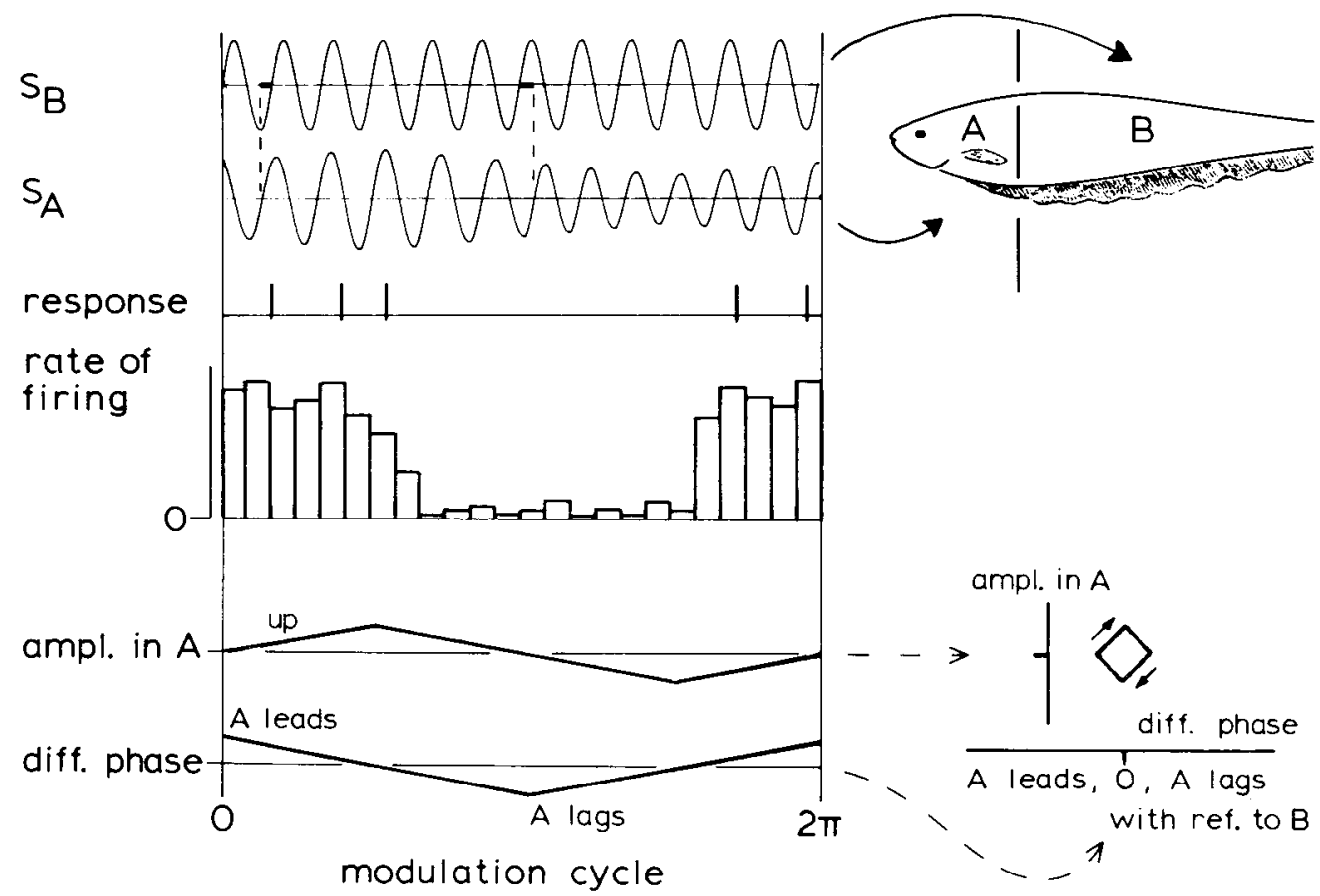

Figure 1. Schematic presentation of two-chamber stimulation regimen. A pure sine wave stimulus $\left(S_{B}\right)$ is applied to body region $B$ and a modulated sine wave stimulus $\left(S_{A}\right)$ is applied to body region $A$. $\mathrm{S}_{\mathrm{A}}$ is being modulated in amplitude as well as in its phase with reference to $S_{B}$ (differential phase between $A$ and $B$ ). In the particular example given, $S_{A}$ leads $S_{B}$ at the beginning of the cycle (first dashed line) and, therefore, lags $\mathrm{S}_{\mathrm{B}}$ in the second half of the cycle (second dashed line). The center of the diagram indicates spike responses of a neuron and a histogram showing the mean rate of firing over many stimulus modulation cycles. The inset in the lower right shows the form of modulation in a two-dimensional, amplitude-phase plane. The clockwise sense of rotation corresponds to a negative difference frequency, $D f$, between a foreign signal and the animal's own signal. Sign reversal of the phase modulation also reverses the sense of rotation of the graph. To demonstrate details of the phase modulation, a sine wave pattern was plotted with only 12 cycles/modulation cycle. Note that, for a fish with an EOD frequency of $400 \mathrm{~Hz}$ and a $D f$ of $4 \mathrm{~Hz}$, a total of $100 \mathrm{EOD}$ cycles would fall into one modulation cycle. With the foreign signal having an amplitude $30 \%$ of that of the animal's own EOD, the relative amplitude modulation is $30 \%$, and the phase modulation is approximately 0.05 of the EOD cycle, which amounts to maximal lead and lag times of 125 $\mu$ sec for a $400-\mathrm{Hz}$ EOD.

Df (see review in Heiligenberg, 1980). In order to present modulations in stimulus amplitude and modulations in stimulus phase independently, we placed the animal into a chamber with a partition fitted around the fish's pectoral region so that separate and independent electrical stimulus regimens could be applied to the head and to the tail region. By using electronic methods applied in earlier studies (Heiligenberg and Bastian, 1980), we were able to modulate the amplitude and phase of a sinusoidal carrier in any manner and thus to present any form of amplitude modulation to either head or tail alone, as well as to control differential phase modulations between head and tail. Commonly, we presented modulations with rhomboid rather than circular graphs in the amplitude-phase plane. Both forms of modulations are equally effective in eliciting JARs. Whereas circular graphs are generated by sinusoidal modulations of amplitude and phase, rhomboid graphs are generated by triangular modulations (see inset in lower right of Fig. 1).

The responses of individual neurons to such stimulus regimens were evaluated in either of two ways: (1) histograms of spike activity were plotted as a function of the modulation cycle (see example in Fig. 1), or (2) while stimulus phase between head and tail was being modulated, the occurrence of individual spikes was plotted both with reference to the current phase difference, $\tau$, between the modulated carrier and the unmodulated carrier, as well as with reference to their phase, $\sigma$, within the cycle of the unmodulated carrier (see Fig. 4).

The electrical separation of the two compartments was approximately $40 \mathrm{~dB}$; i.e., a stimulus presented to one of the two compartments alone, with an intensity of $1 \mathrm{mV} / \mathrm{cm}$, measured perpendicularly and closely to the animal's body surface, yielded a stimulus of less than $0.01 \mathrm{mV} / \mathrm{cm}$ on the body surface in the other compartment. The abundance of neurons, from the level of primary afferents to higher-order neurons in the torus, which could only be driven through one compartment, convinced us that this degree of stimulus separation was sufficient.

Electrosensory afferents to the torus semicircularis: Amplitude and phase information required for the control of the JAR. The afferent and efferent organization of the torus semicircularis has been studied in great detail using various neuroanatomical techniques (Carr et al., 1981; Scheich and Ebbesson, 1981; Maler et al., 1982). 'I'he pattern of electrosensory afferents, as revealed by anterograde and retrograde labeling with extracellularly injected HRP, is summarized in Figure 2.

The ELLL, termination site of primary electroreceptive afferents and formerly called posterior lateral line lobe (Maler et al., 1981; Bullock and Northcutt, 1982), projects, via the lateral lemniscus, predominantly to the contralateral torus. The four horizontally separated electrosensory maps of the ELLL (Heiligenberg and Dye, 1982; Carr et al., 1982) merge into one map in the torus, which consists of 12 laminae (Fig. 2, see insert).

The spherical cells of the ELLL (Fig. 2, solid circle), which are driven by T-type electrosensory afferents, fire one spike per EOD cycle. This spike is phase-locked to the zero-crossing of the EOD signal within the receptive field on the body surface, and the difference in the timing of spikes in two spherical cells thus reflects the phase difference of the electrical signal in their respective receptive fields. Extracellular and intracellular injec- 

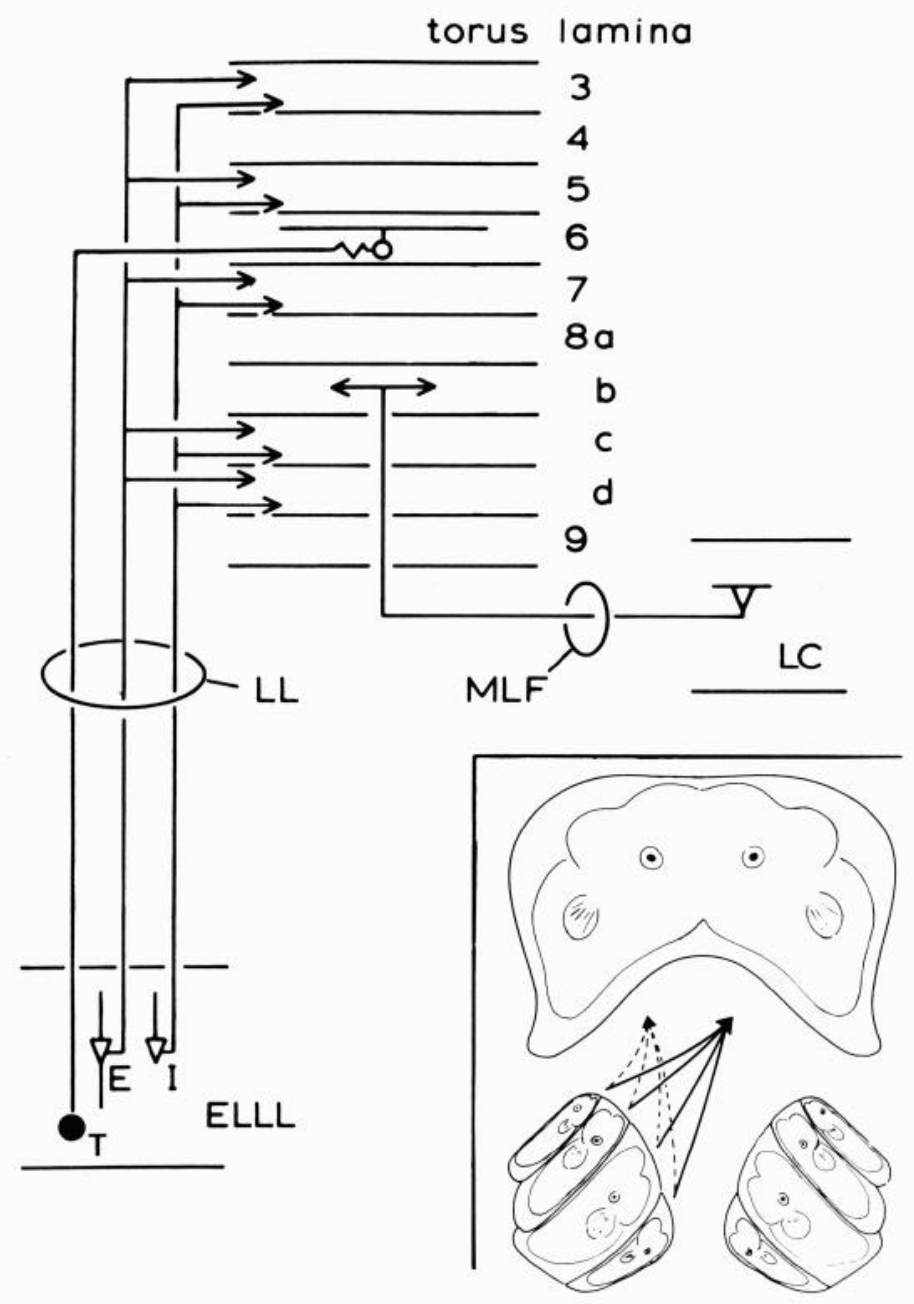

Figure 2. Electrosensory afferents to the torus semicircularis. Two types of pyramidal cells of the ELLL, E- and I-units, relay information about amplitude modulations to laminae $3,5,7,8 \mathrm{c}$, and $8 \mathrm{~d}$ via the lateral lemniscus $(L L)$. The spherical cells of the ELLL $(T$, solid circle) relay phase information electrotonically to the giant cells in lamina 6 (open circle). The significance of the projections of eurydendroid cells of the lobus caudalis $(L C)$ to lamina $8 \mathrm{~b}$ via the medial longitudinal fasciculus $(M L F)$ is still not known. The inset in the lower right shows a horizontal section through the torus (top) and the two ELLLs (bottom). The four maps of the body represented in the ELLL, with the left side of the body in the left ELLL, project predominantly to the contralateral side of the torus.

tions of HRP and Lucifer Yellow have shown that the spherical cells project in a somatotopic manner exclusively to lamina 6 of the torus. Current ultrastructural and physiological studies by Carr et al. (1983) show that the axons of these spherical cells contact the giant cells of lamina 6 via gap junctions. The giant cells of lamina 6 fire one spike per EOD cycle, phaselocked to the cycle, as do the spherical cells of the ELLL.

Two types of pyramidal cells in the ELLL, basilar pyramidal cells, or E-units, and non-basilar pyramidal cells, or I-units, receive $\mathrm{P}$-type receptor input and thus respond to local amplitude modulations of the EOD on the animal's body surface. Eunits fire in response to an increase in stimulus amplitude, and I-units fire in response to a decrease in stimulus amplitude (Bastian and Heiligenberg 1980a; Bastian 1981a, b; Saunders and Bastian, 1984). Gross injections of HRP have shown that both sets of pyramidal cells project to laminae $3,5,7,8 \mathrm{c}$, and $8 \mathrm{~d}$, but they have failed to reveal the patterns of projections of individual cells (Carr et al., 1981; Maler et al., 1982). We labeled a total of nine pyramidal cell projections, four of the I-type and five of the E-type, by intracellular injections into their axonal terminations in the torus. All pyramidal cell afferents projected to more than one lamina, but they differed in the size of their terminal fields within given laminae. For example, whereas some afferents projected heavily to lamina 7 , others passed lamina 7 altogether (see examples in Fig. 3, $a$ and $b$ ). Unfortunately, our injections were not sufficient to label these pyramidal cells up to the level of their somata in the ELLL. However, since E- and I-units are the only cells of the ELLL, besides spherical cells, which project to the torus, and since these cells show characteristic and distinct responses to amplitude modulations (Saunders and Bastian, 1984), we can establish their anatomical identity.

The lobus caudalis of the cerebellum projects, via the medial longitudinal fasciculus, to the contralateral lamina $8 \mathrm{~b}$ of the torus (Carr et al., 1981). Nothing is known about the functional role of this projection. We labeled only two afferents from the lobus caudalis (Fig. $3 c$ ) and found that they were marginally driven by amplitude modulations. Our stimulus regimens appeared to be inappropriate for these neurons.

The torus receives all electrosensory information necessary for the control of the JAR through afferents from the ELLL. E- and I-units report local rises and falls of stimulus amplitude, and differential phase (i.e., the difference in the timing of zerocrossings of stimuli in two parts of the body surface) is reflected by the difference in timing of spikes of spherical cell afferents assigned to these parts of the body surface. The JAR requires that local amplitude information is evaluated in reference to differential phase information. The fish must determine whether a local rise or fall in stimulus amplitude coincides with a local stimulus phase lag or lead (see review in Heiligenberg, $1980,1983)$. The computation of differential phase and local amplitude modulations is executed by various cell types in different laminae of the torus which will be described in the following sections.

The computation of differential phase. Each giant cell of lamina 6 sends extensive axonal branches to ipsilateral and contralateral areas of this lamina and thus relays phase information from its own receptive field to parts of this lamina which receive phase information from more distant receptive fields (Carr et al., 1983). The somatotopic order of spherical cell afferents to lamina 6 ensures that the timing, $t_{A}$, of afferent spikes within a given area reflects stimulus phase in the associated receptive field, $A$, of the body surface. The timing, $t_{B}$, of spikes in a passing axon of a giant cell reflects stimulus phase in some other receptive field, $B$. Therefore, a comparison of $t_{A}$ and $t_{B}$ should yield a measure of the differential phase, $t_{A}-t_{B}$. Anatomical studies by Carr et al. (1983) have identified small cells within lamina 6 which are contacted by giant cell axons. These small cells were found in the present study to discriminate differential phase.

By applying the stimulus regimen outlined in Figure $4 a$, we found that these small cells follow modulations in differential phase between head and tail. The neuron presented in Figure $4 b$, for example, is silent if the zero-crossing of the stimulus in the head region precedes the zero-crossing of the stimulus in the tail region, i.e., for small negative values of the phase difference, $\tau$. The neuron is activated for small positive values of $\tau$. These small cells did not respond to modulations of stimulus amplitude in either compartment (Fig. 5), provided that such modulations remained within a naturally occurring range of approximately $30 \%$ of the carrier amplitude (note that for much larger modulations, T-afferents fire only intermittently at the minimum of the modulation cycle). We labeled a total of four small cells which could be driven by the stimulus regimen in Figure $4 a$. Other small cells in lamina 6 which could not be driven by this regimen presumably compared parts of 
(a)

\section{I-afferent (C)}

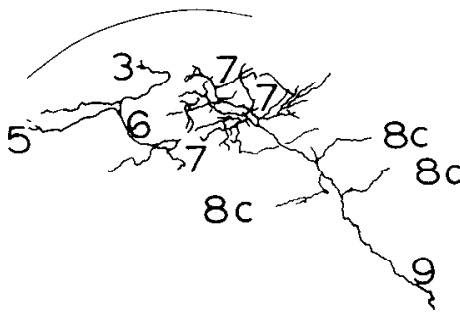

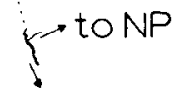

to ELLL

\section{I-afferent}
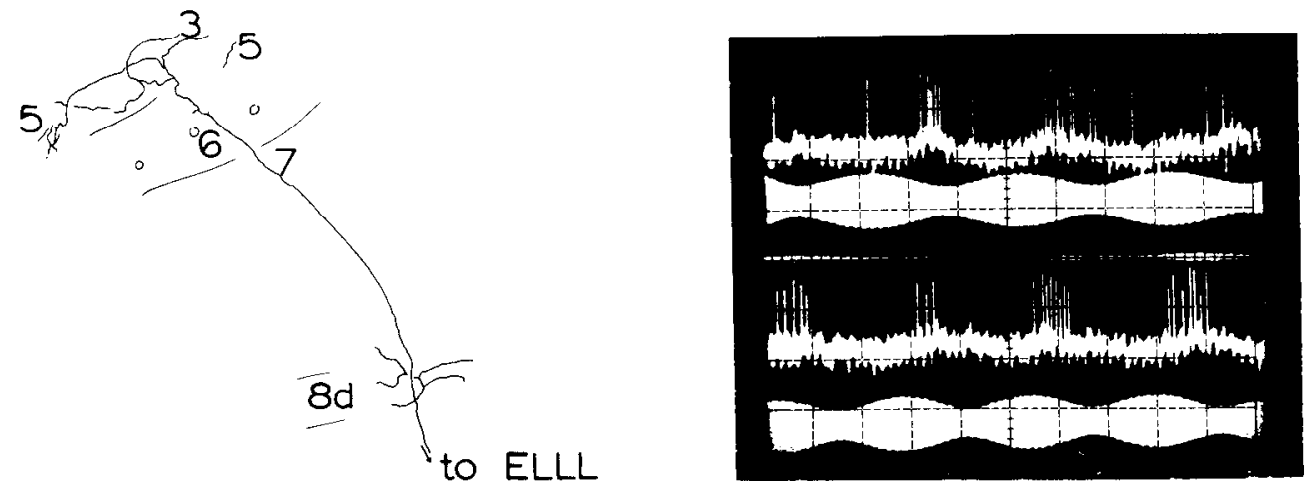

Figure 3. Electrosensory afferents to the torus. $a$, I-type afferent from ELLL, with collateral to nucleus prae-eminentialis (NP). Numbers indicate the identity of the local lamina. The pyramidal cell afferents penetrate lamina 6 via columns of vertical neuropil (Carr et al., 1981). $b$, I-type afferent without terminals in lamina 7 . The same variation in terminal pattern is observed in E-type afferents. $c$, Eurydendroid afferent from lobus caudalis $(L C)$. The terminals are limited to lamina $8 \mathrm{~b}$. The unit is weakly driven by amplitude modulations. The inset in the lower right shows a recording of the cell in $a$ responding to a beat pattern of positive (top) and negative (bottom) Df. Regardless of the sign of $D f$, the neuron fires in response to decreasing stimulus amplitude. the body surface, such as right versus left, which could not be stimulated separately by our stimulus regimen.

Small cells may be phase sensitive by virtue of an inhibitory mechanism. The cell in Figure $4 b$ is almost silent if the zerocrossing of the signal in the head region briefly precedes or coincides with the zero-crossing of the signal in the tail region. The cell in Figure $4 c$ is silent for a certain phase lag. An inhibitory process is also suggested by the data shown in Figure $6 a$ : this unit is silent whenever the zero-crossing of the signal at the tail precedes that of the signal at the head. With the stationary signal in the head region turned off, however, the unit fires tonically and at a higher mean rate $(55 / 5$ versus 63 / 10). The phase modulation of a sinusoidal signal necessarily entails a modulation of its instantaneous frequency (the inverse of the interval between successive zero-crossings), regardless of the presence of a stationary reference signal in the other region of the body. The fact that this neuron does not follow the modulation cycle in the absence of the reference signal shows that it is not driven by modulations in instantaneous frequency.

The standard modulation rate in our experiments was $2 \mathrm{~Hz}$, which is sufficiently fast to drive the JAR but yet slow enough to allow for immediate detection of neuronal responses by the experimenter. Since earlier behavioral experiments (Heiligenberg and Bastian, 1980) had given evidence that animals can discriminate stationary phase differences, we tested small cells by presenting them with phase modulations at the extremely slow scan rate of $0.2 \mathrm{~Hz}$ and with constant phase differences maintained for at least $3 \mathrm{sec}$. The data in Figure $6 b$ demonstrate that small cells are able to discriminate stationary phase differences. Consistent with the data for the normal modulation rate shown in Figure $6 a$, this cell fires when the signal in the tail compartment lags the signal in the head compartment for slow modulation rates and fixed phase differences.

The scattergrams in Figure 4 indicate moderate phase coupling of spikes either to the $S_{A}$ cycle (Fig. $4 b$ ) or to the $S_{B}$ cycle (Fig. 4c). This phase coupling demonstrates that small cells are synaptically close to $\mathrm{T}$-afferents and/or giant cells, which both show very strict phase coupling. That we found, within the same head region of lamina 6 , small cells that were silenced by either a phase lead or a phase lag of the signal in the head compartment with reference to that in the tail compartment suggests the existence of at least two classes of small cells. This physiological difference may be related to morphological differences of small cells observed in ultrastructural and Golgi studies (Carr et al., 1983; C. E. Carr and L. Maler, submitted for publication).

According to studies by Carr et al. (1983), the small cells of lamina 6 project out of this lamina. Although we do not know the cellular connections within deeper laminae, we have found physiological evidence that various cell types, notably in lamina 8 , must receive this phase information from lamina 6 . The responses of these cells to phase and amplitude information will be described in the following section.

The convergence of phase and amplitude information in individual cells. We have identified 38 cells in deeper laminae of the torus which responded to modulations of stimulus amplitude to various degrees but were predominantly driven by modulations in phase. An example is shown in Figure 7. This cell is excited by a phase lag of head versus tail, which is equivalent to a phase lead of tail versus head. Similar histograms are therefore obtained in Figure 7, $a-a$ and $a-e$. Concur rent modulations in stimulus amplitude have negligible addi- 

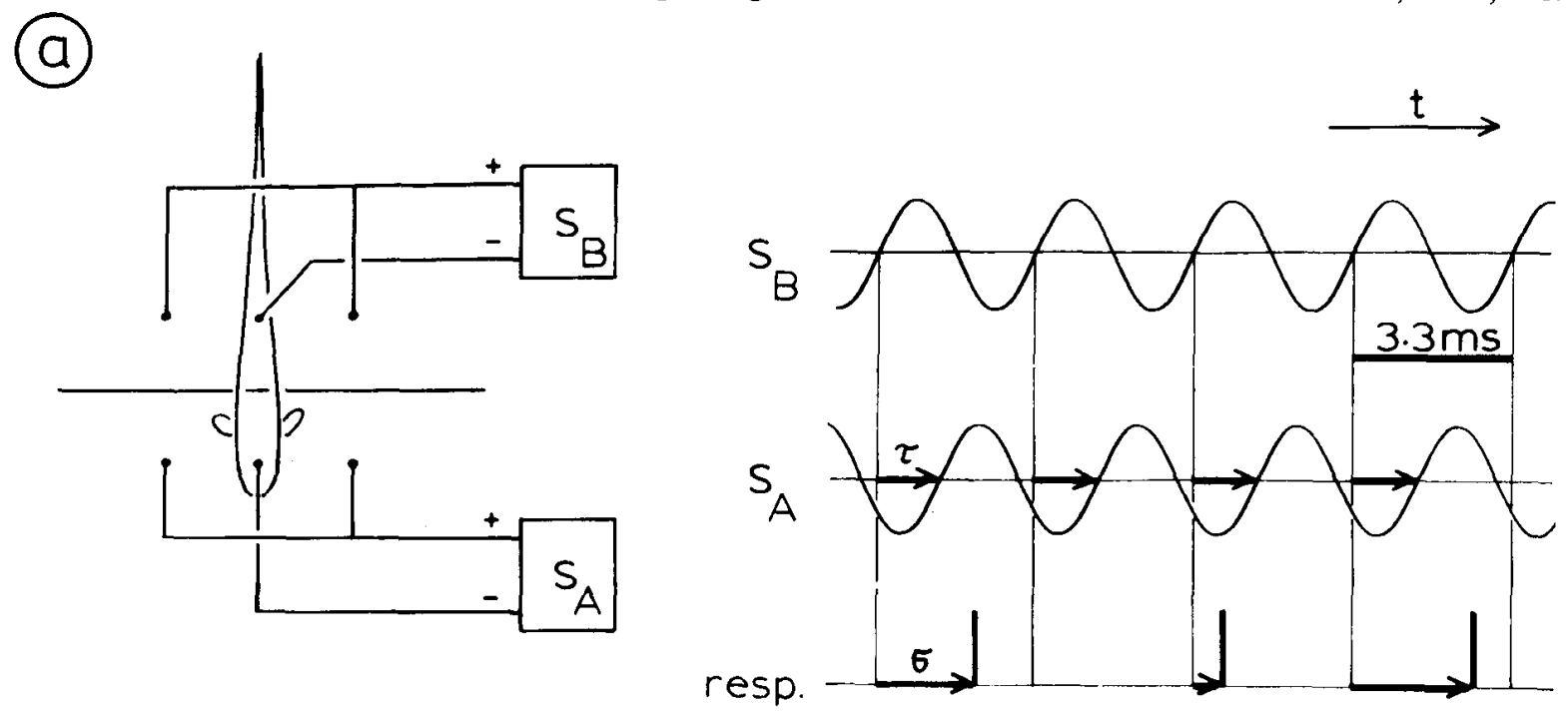

(b)

$\tau$ modulated at $2 \mathrm{~Hz}$, triang.

small cell, Iam, 6 (9 Aug 83)
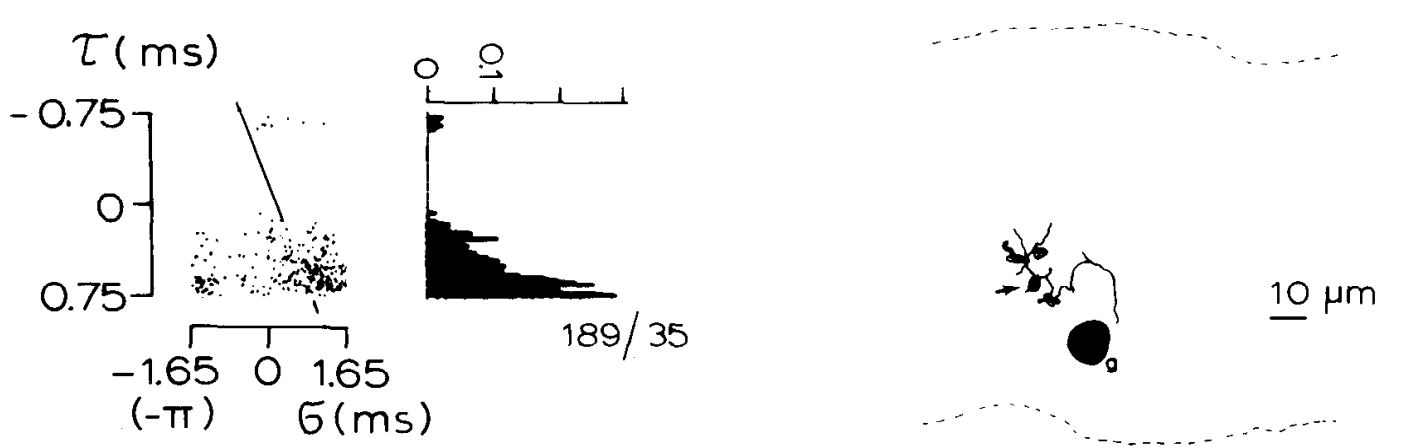

(C)

small cell, Iam. 6 (10 Aug 83)
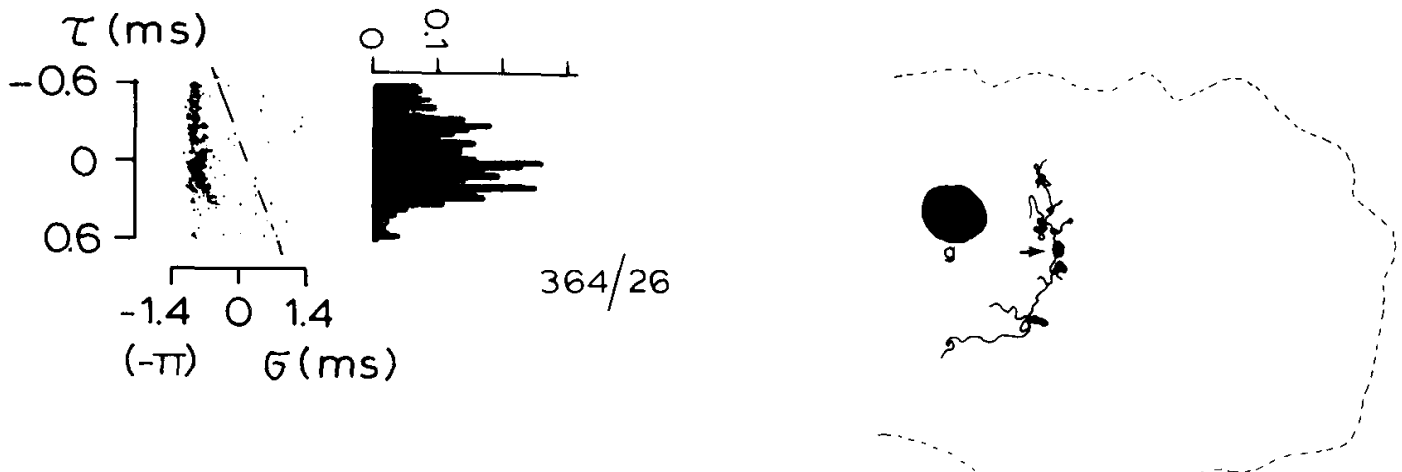

Figure 4. Responses of two small cells in lamina 6 to modulations of differential phase between head and tail. $a$, Schematic representation of stimulus regimen with definition of differential phase $(\tau)$ and latency $(\sigma)$ of neuronal response. The phase $(\tau)$ is modulated by a triangular wave form at a rate of $2 \mathrm{~Hz}$ between the extremes denoted in $b$ and $c$. A positive $\tau$ represents a phase lag of the head signal with respect to the tail signal. $b$, The occurrence of spikes is plotted as a function of $\tau$ and $\sigma$ in the scatter diagram on the left. The diagonal line indicates the timing ( $\tau$ ) of the zero-crossing of $S_{A}$ with respect to the cycle of $S_{B}$. A neuron firing phase-locked to $S_{A}$ would yield a distribution of dots parallel to this diagonal line. A neuron firing phase-locked to $\mathrm{S}_{\mathrm{B}}$ would yield a distribution of dots along a vertical line. The histogram to the right gives the rate of firing per $10-\mu \mathrm{sec}$ intervals. The notation underneath gives total number of spikes per total number of phase modulation cycles. A camera lucida drawing of the Lucifer Yellow-filled cell is shown to the right (arrow). The soma of the nearest giant cell ( $g$ ) is shown for size comparison. $c$, Same as $b$ for another neuron. This neuron, in contrast to that in $b$, is silent for a phase lag. Both neurons are from the head region of lamina 6. 

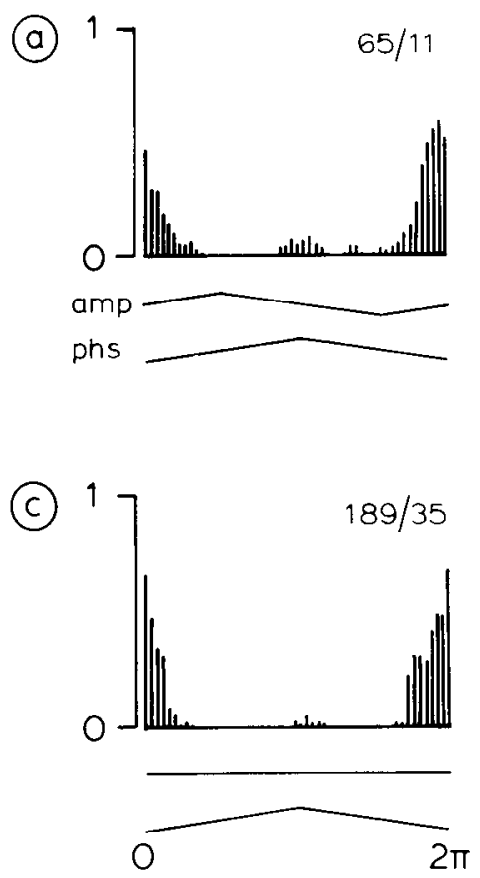
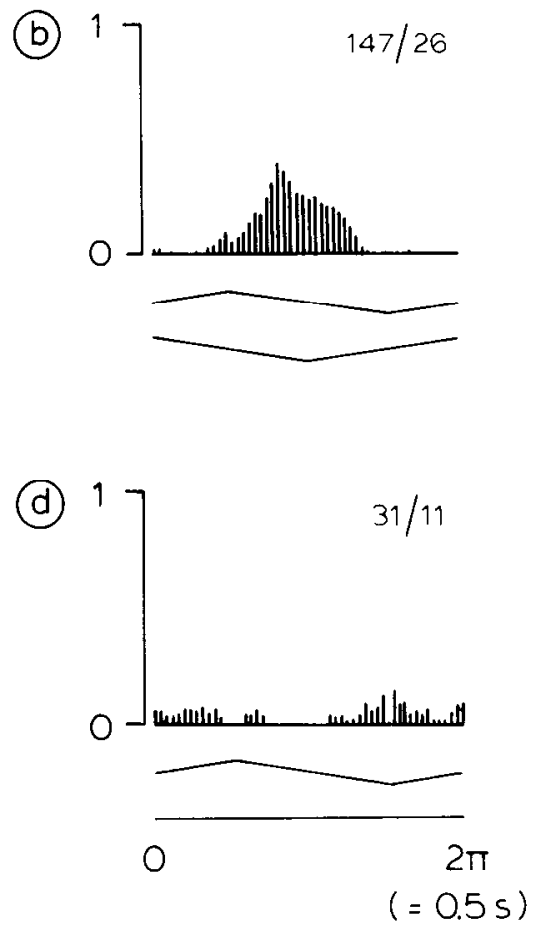

Figure 5. Responses of a small cell (9-Aug-83) in lamina 6 to modulations of amplitude and phase applied to the head region with phase referenced to the tail. The histograms, plotted as a function of the modulation cycle $(2 \mathrm{~Hz})$, show the meun rate of firing per successive 10 -msec bins. The form of stimulation is shown under each histogram as indicated in Figure 1 ; i.e., an upward excursion in the amplitude characteristic indicates a rise in amplitude, and an upward excursion in the phase characteristic indicates a shift from phase lag to phase lead. The notation in the upper right gives total number of spikes over total number of modulation cycles. The comparison of a and $c$ shows that this unit only responds to modulations in differential phase between head and tail. It is largely silent with phase synchrony and phase lead and is insensitive to modulations of amplitude alone (d). All histograms were smoothed by a gliding weight function with the weight ratios, 1:2:4:2:1. tional effects, so that the shapes of the histograms in Figure 7, $a-b$ and $a-c$, as well as in $a-f$ and $a-g$, can be predicted from those in Figure 7, $a-a$ and $a-e$. Since the histograms $a-b$ and $a-$ $c$ are based upon identical amplitude modulations but opposite phase modulations, their stimulus regimens reflect graphs with opposite senses of rotations, i.e., Dfs of opposite signs. Since the shape of a beat envelope is determined by the amplitude modulation alone, spike histograms plotted in reference to the envelope or amplitude modulation cycle (as in Fig. 7, $a-b$ and $a-c)$ are $D f$-sign dependent if the neuron responds to modulations in phase. The cell in Figure 7 therefore is considered " $D f$ sign sensitive" in the sense of earlier studies (Bastian and Heiligenberg, 1980b; Partridge et al., 1981).

Most importantly, the response of the unit in Figure 7 does not depend upon which particular region of the body, head or tail, is phase-modulated with respect to the other, since only the difference in the timing of zero-crossings between the stimuli in the two regions is computed. This cell is also able to discriminate stationary phase values (Fig. $7 b$ ) as clearly as do small cells of lamina 6 .

The discrimination of stationary phase values was taken as a diagnostic tool to ensure that the phase sensitivity of a cell was based upon afferent information supplied by the lamina 6 system. We frequently found cells which could be driven by amplitude modulations applied to either region of the body, head or tail. Such cells also commonly responded at least weakly to pure phase difference modulations between these parts of the body as long as such modulations occurred at a sufficiently high rate, such as $2 \mathrm{~Hz}$. These units, however, failed to respond to phase modulations as slow as $0.2 \mathrm{~Hz}$ and did not discriminate stationary differences in phase. Several of these cells were pyramidal cell afferents, many of them recorded directly in the ELLL, whose receptive fields extended into both compartments of the chamber. Based upon the description of pyramidal cells of the ELLL and their afferent organization by Maler et al.
(1981), one can predict that such cells should be driven by sufficiently strong modulations of differential phase within their receptive field. The ensuing synchronization and desynchronization of $\mathrm{P}$-receptor afferents from separate compartments should mimic a modulation in stimulus amplitude. These ELLL cells are therefore pseudo-phase sensitive, seeing the scan in phase difference as an amplitude modulation, and this artifactual response is communicated to higher-order neurons. For this reason, we excluded all cells from our analysis which responded to amplitude modulations in each compartment of the chamber, and we categorize as "phase sensitive" only those cells which responded to phase modulations with this restriction.

The majority of phase-sensitive cells in the deeper laminae of the torus also responded significantly to modulations of stimulus amplitude, and the joint presentation of phase and amplitude modulations resulted in a superposition of the responses of the cells to phase and amplitude modulations alone. An example is given in Figure 8 . This cell is excited by a rise in stimulus amplitude in the tail region as well as by a phase lead in the tail region with reference to the head. Consequently, the activity of this cell is entrained most strongly under that particular joint modulation of amplitude and phase which corresponds to a clockwise sense of rotation, i.e. a negative $D f$ applied to the tail region (Fig. $8 g$ ). The same regimen applied to the head region (Fig. 8, $d$ and $e$ ) yields responses of similar strength to either sense of rotation as the amplitude component has no effect in the head region. The receptive field of this cell could be seen as consisting of three components, one component being a P-receptor input from the tail, the other two components being $\mathrm{T}$-receptor inputs from head and tail, respectively, supplying information about differential phase between these two parts of the body surface. Much as in the case of the neuron presented in Figure 7 , this cell is also considered $D f$-sign 
(a)

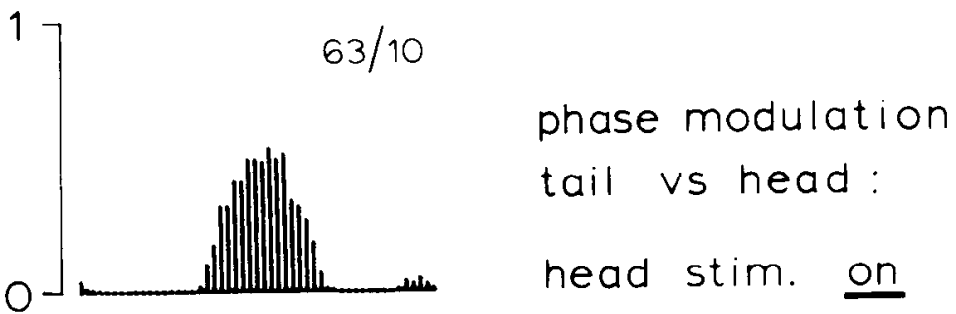

Figure 6. Responses of a small cell of lamina 6 to differential phase. $a, A$ modulation of phase between approximately + and $-\pi / 4$, in the form of a triangular modulation wave form and at a rate of $2 \mathrm{~Hz}$, shows that the unit is silent if the tail leads the head (top diagram). The unit becomes active if the stimulus to the head is turned off (bottom diagram), and it fires throughout the modulation cycle. The data are presented in the format of Figure 5. b, The same cell responds to modulations of phase 10 times slower than those in $a$ and also discriminates stationary phase values. In the upper diagram, phase was modulated at a rate of $0.2 \mathrm{~Hz}$ over a period of $14 \mathrm{sec}$, and a total of 117 spikes were counted. The ordinate shows mean number of spikes per phase bin, the width of the bin being the equivalent of $10 \mu \mathrm{sec}$ ( $2 \pi$ corresponds to $3.22 \mathrm{msec}$ in this fish). The lower diagram shows firing frequen. cies measured while a given phase value was kept constant for at least 3 sec. A positive phase value represents a phase lag.

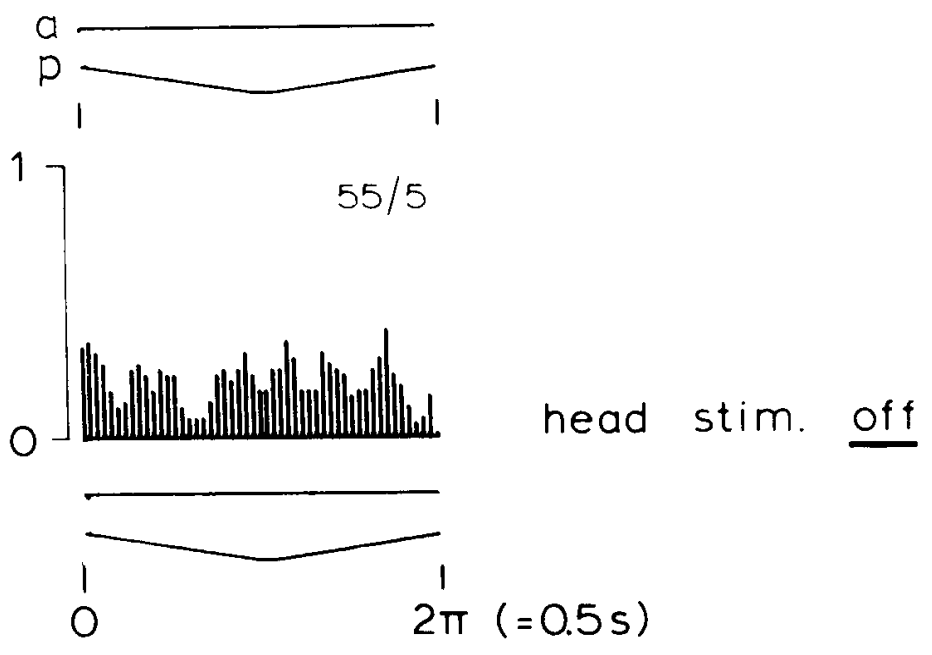

(b)
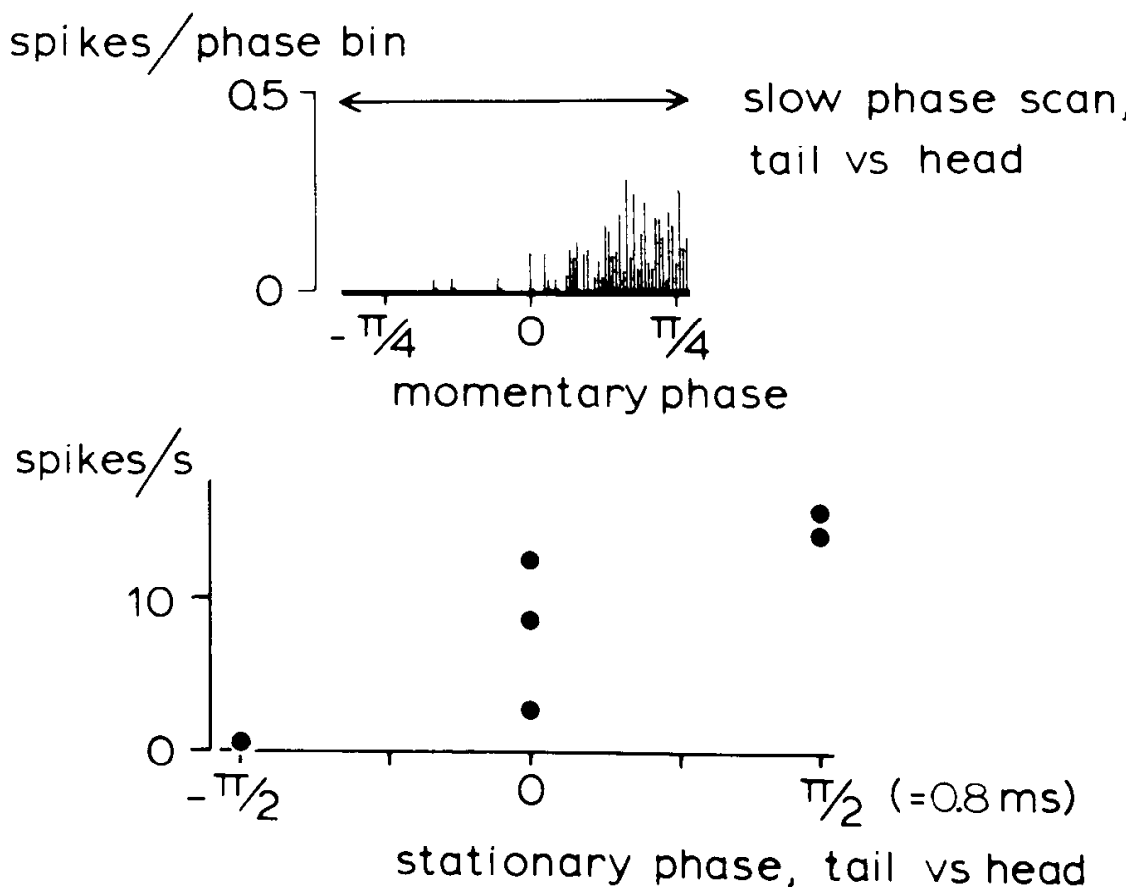

sensitive since histograms plotted as functions of the amplitude modulation cycle are $D f$-sign dependent.

The analysis of the few small cells identified in lamina 6 showed that units within the rostral part of the torus, i.e., the area of $\mathrm{T}$-afferents from the head, were inhibited either by a phase lead or a phase lag of the head with respect to the tail (cf. Fig. 4, $b$ and $c$ ). This difference in response to phase is also observed in phase-sensitive neurons of the deeper laminae, and those cells which are excited by modulations in amplitude also are either excited by a phase lead or a phase lag in the same compartment. This difference allows us to distinguish four classes of cells, first, according to their response to amplitude modulations, i.e., E- or I-type, and, second, according to their preference of either a phase lead or a phase lag in their specific, amplitude-sensitive region of the body.

Of a total of 38 phase-sensitive cells located below lamina 6 , 


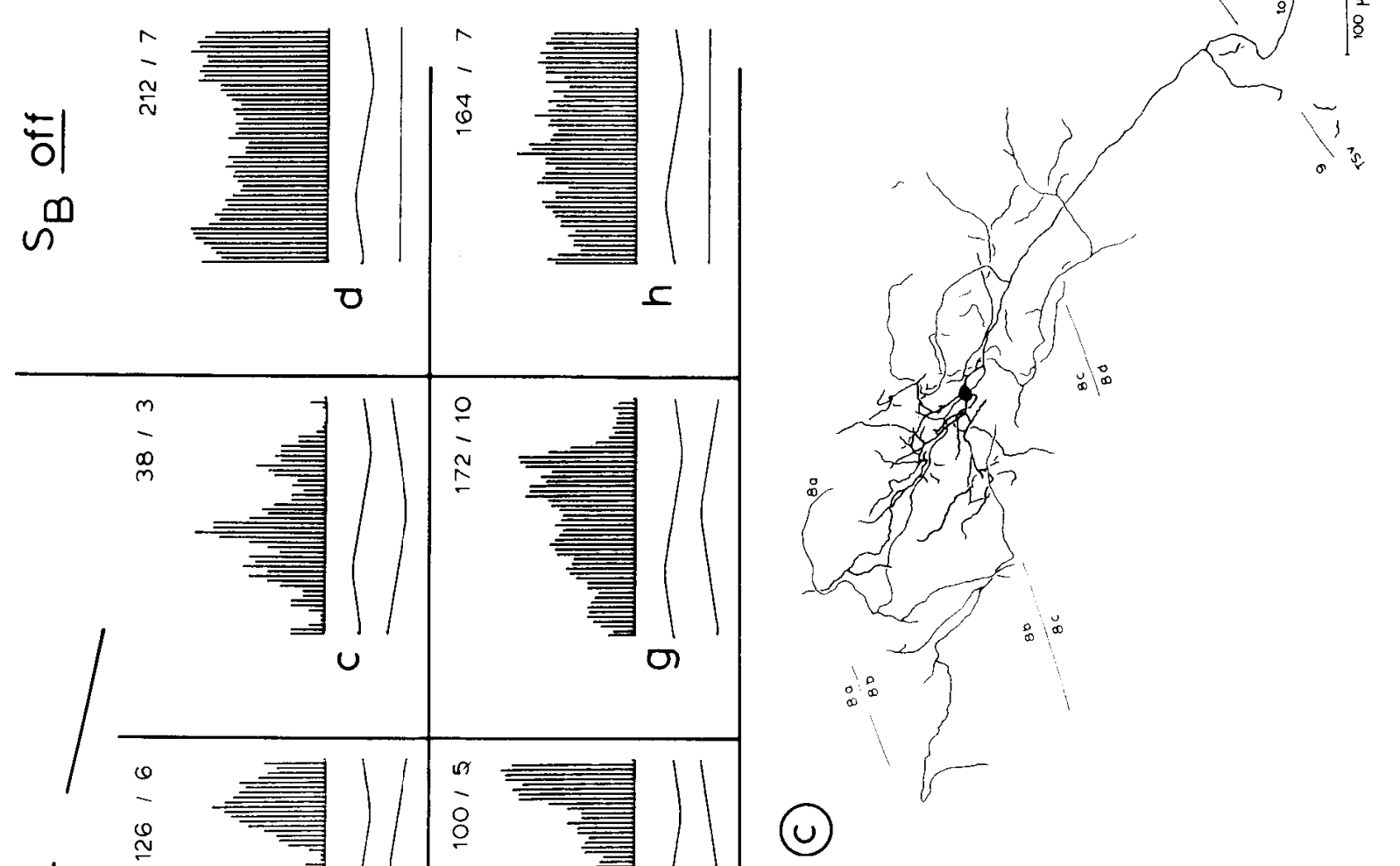

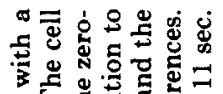

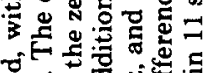

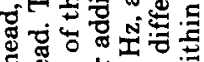

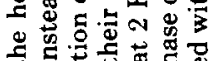

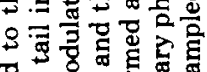

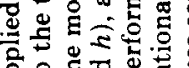

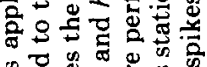

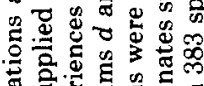

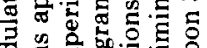

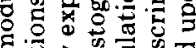

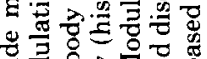

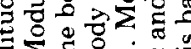

过 8 के

究

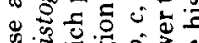

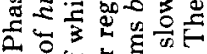

उ

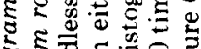

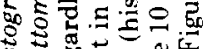

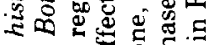

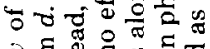

จ

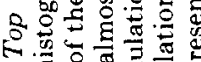

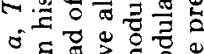

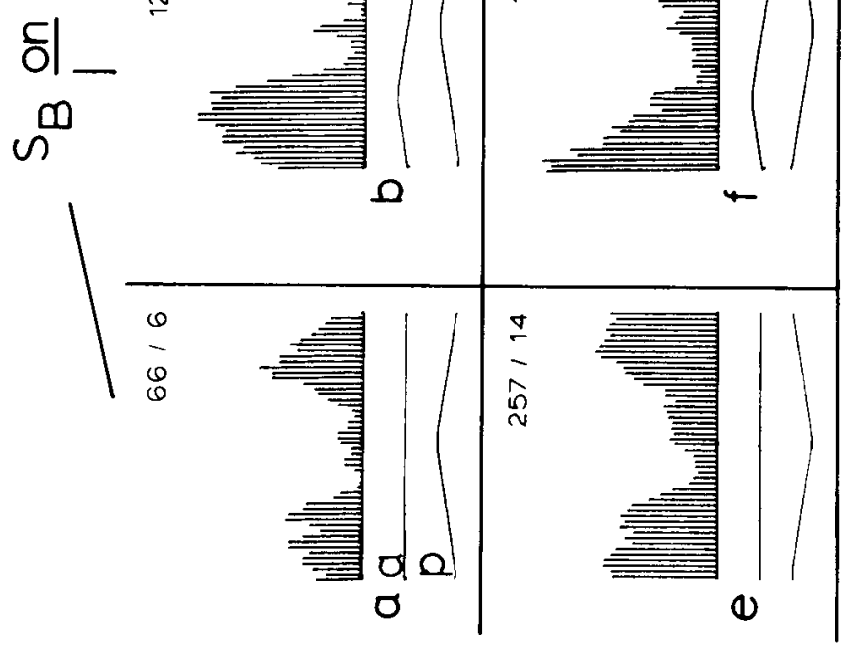

点

(0) $\sim^{\frac{1}{5}}$
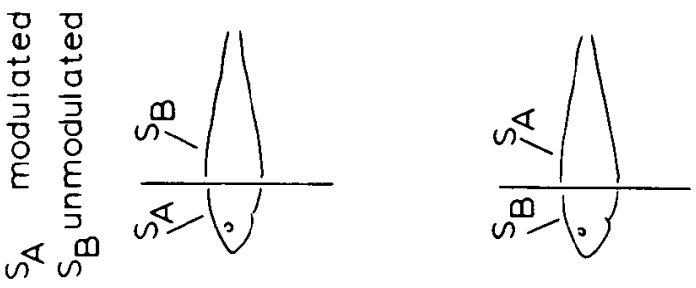

(อ)

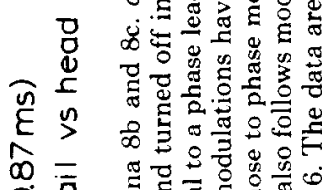

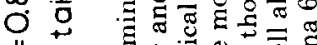

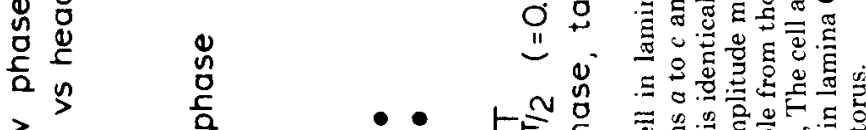

$\frac{3}{n}=$

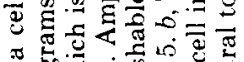

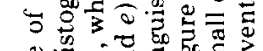

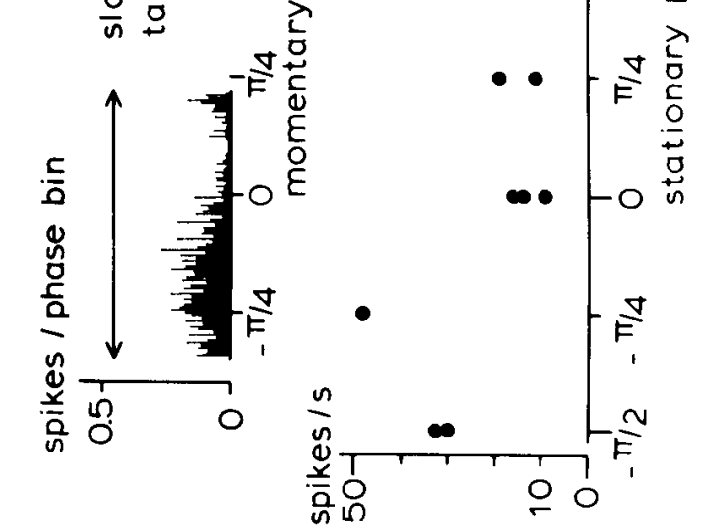

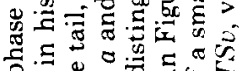

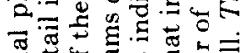

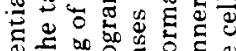

屯.

च 0

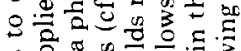

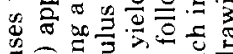

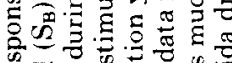

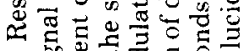

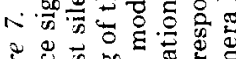

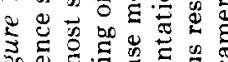




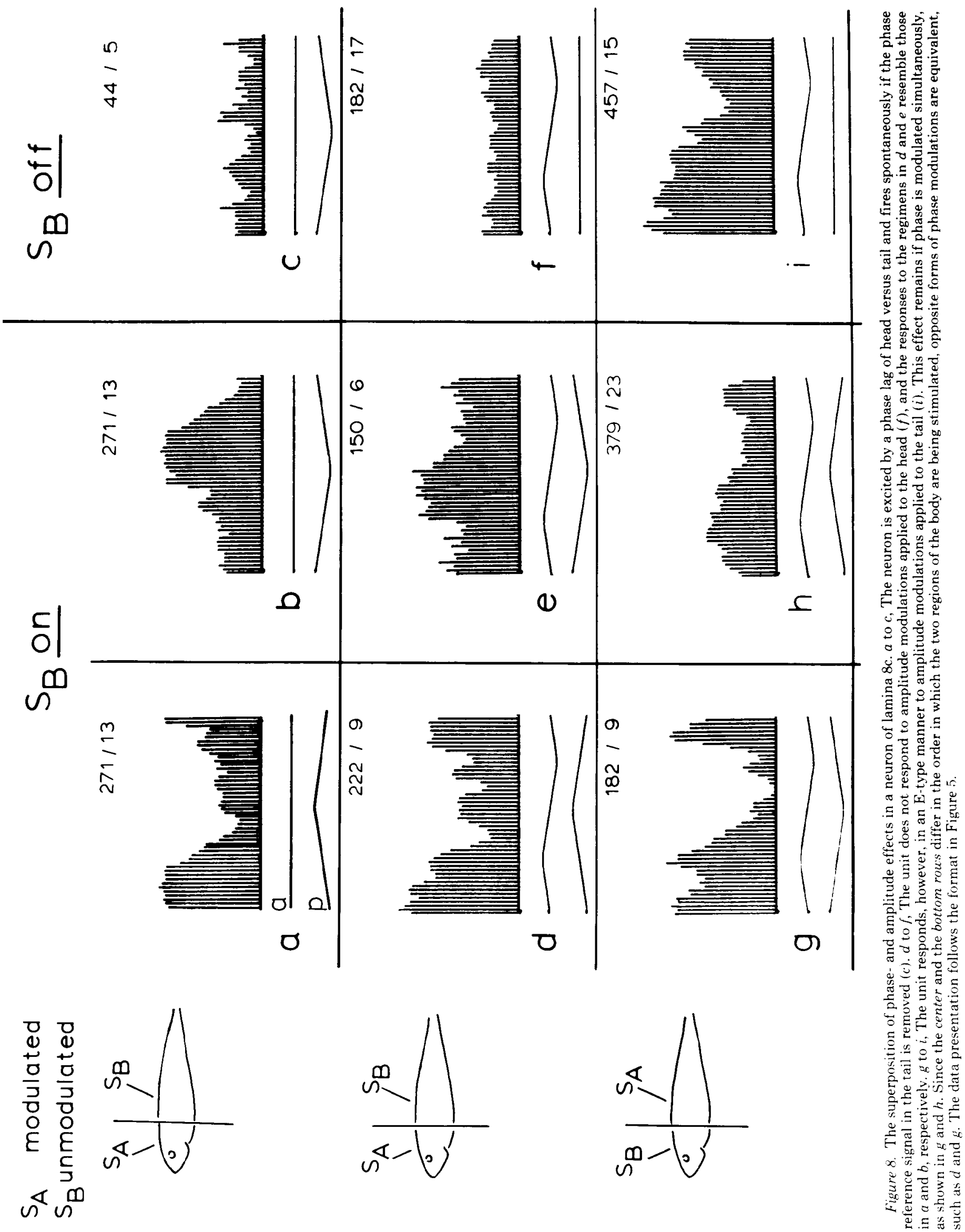




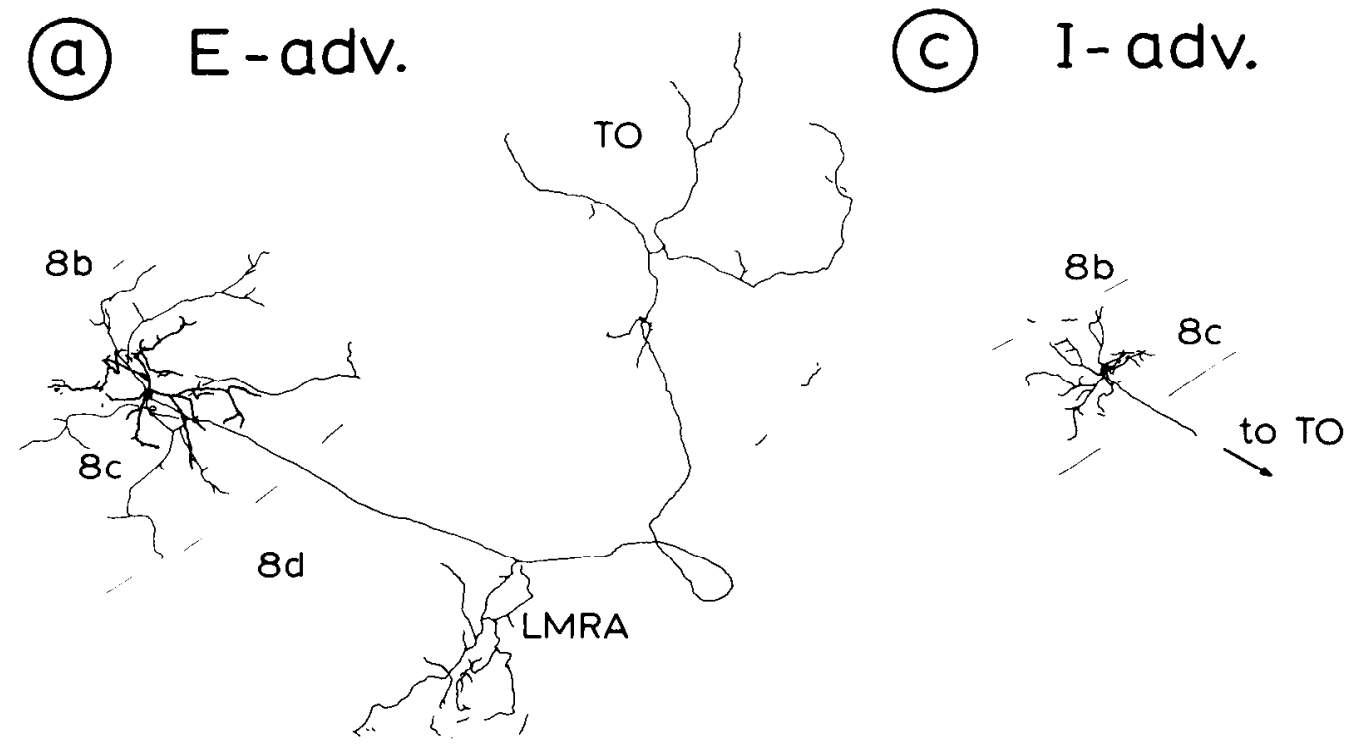

Figure 9. Cells in deeper laminae of the torus ( $8 b$ and $8 c$ ) which respond to modulations of amplitude as well as to modulations of phase. Responses to amplitude are in the manner of the $E$ - or the I-type, and the cell is excited either by a local phase advance or a local phase delay. All cells in this figure project to the tectum (TO), and the more heavily labeled cells in $a$ and $b$ also show collaterals in the lateral mesencephalic reticular area (LMRA). The numbers label laminae of the torus.
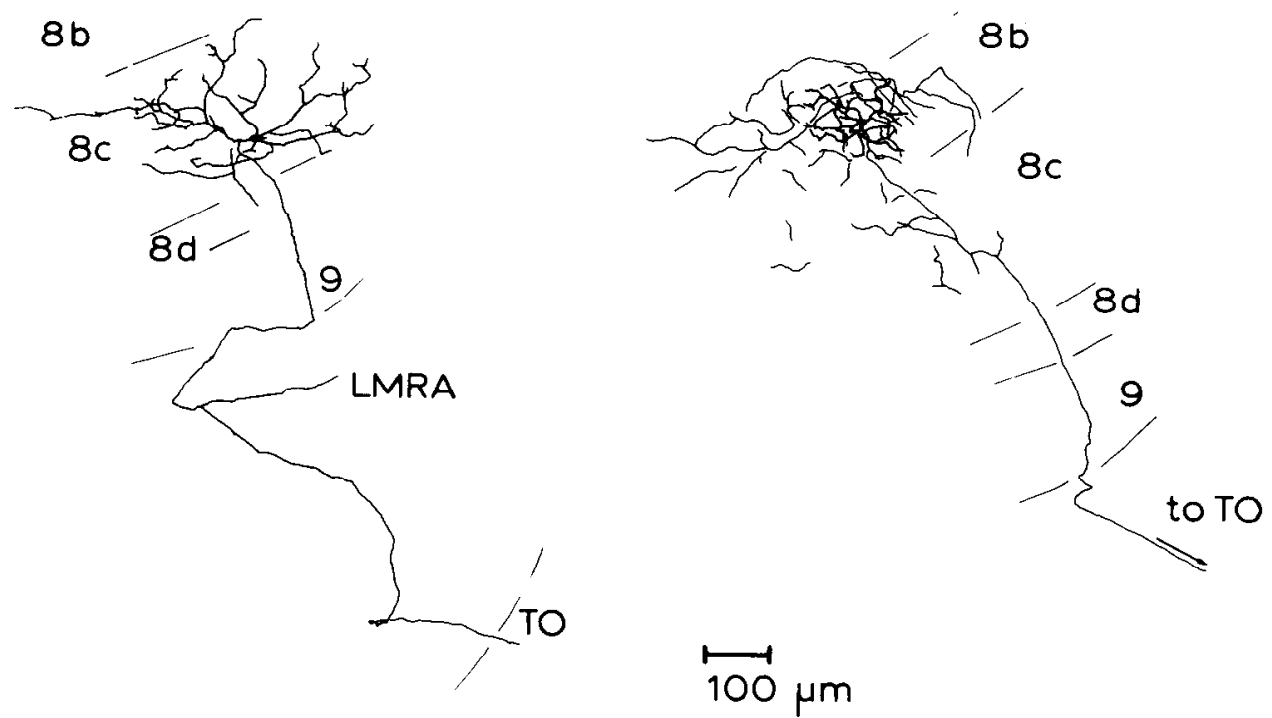

only 4 failed to respond to amplitude modulations. Of the remaining 34 cells, 22 were of the E-type, and 12 were of the Itype. Of the 22 E-type units, 8 were excited by a phase advance in the amplitude-sensitive region of the body, whereas 13 were excited by a phase lag. The corresponding figures for the I-type units are 5 and 6 . One E-type and one I-type unit could not be classified in this manner since they were excited by large phase lags as well as phase leads $(\sim \pm \pi / 4)$.

The coding of amplitude and phase information by cells projecting to the optic tectum. We found that representatives of all four physiological classes of amplitude- and phase-sensitive cells, E-lead, E-lag, I-lead, and I-lag, project to the optic tectum. An example of the responses of the E-lead type is shown in Fig. 8. Camera lucida drawings of representatives of these four classes of cells are shown in Figure 9.

Many cells of the torus did not respond to modulations in differential phase in our two-chamber paradigm. In any individual instance, one could have argued that this regimen failed to modulate the phase difference between the particular two areas sampled by this cell. (Note that we could not modulate phase differences between the two sides of the body in these experiments. Also, it proved impossible to design a chamber which yielded sufficient electrical isolation between the two sides of the body to allow separate, left and right signal modulation.) However, the statistical distribution of phase-sensitive and non-phase-sensitive cells as a function of their laminar location strongly suggests that some laminae, such as 5,7 , and 8a, have few phase-sensitive cell types under any stimulus regimen. Of a total of 15 amplitude- and phase-sensitive cells, 12 were located in laminae $8 \mathrm{~b}$ through 9 , whereas, of a total of 25 cells which only responded to amplitude modulations, only 8 were found in these laminae. As a further test for the presence of cells insensitive to phase, we used a stimulus regimen (Partridge et al., 1981) which allowed us to modulate phase between almost any parts of the body surface, although not in a form uncontaminated by simultaneous modulations of amplitude.

In this regimen the fish was suspended in the middle of a 40 $\mathrm{cm} \times 40 \mathrm{~cm}$ area of an aquarium, and an $S_{1}$ stimulus was supplied between an electrode in the mouth and an electrode near the tip of the tail. The $\mathrm{S}_{2}$ stimulus was presented between any pair of a circular array of external electrodes, the $S_{2}$ stimulus thus straddling the fish in any of various orientations with respect to its body axis: transversely, longitudinally, or diagonally (see Fig. 11 in Partridge et al., 1981). Because, in 


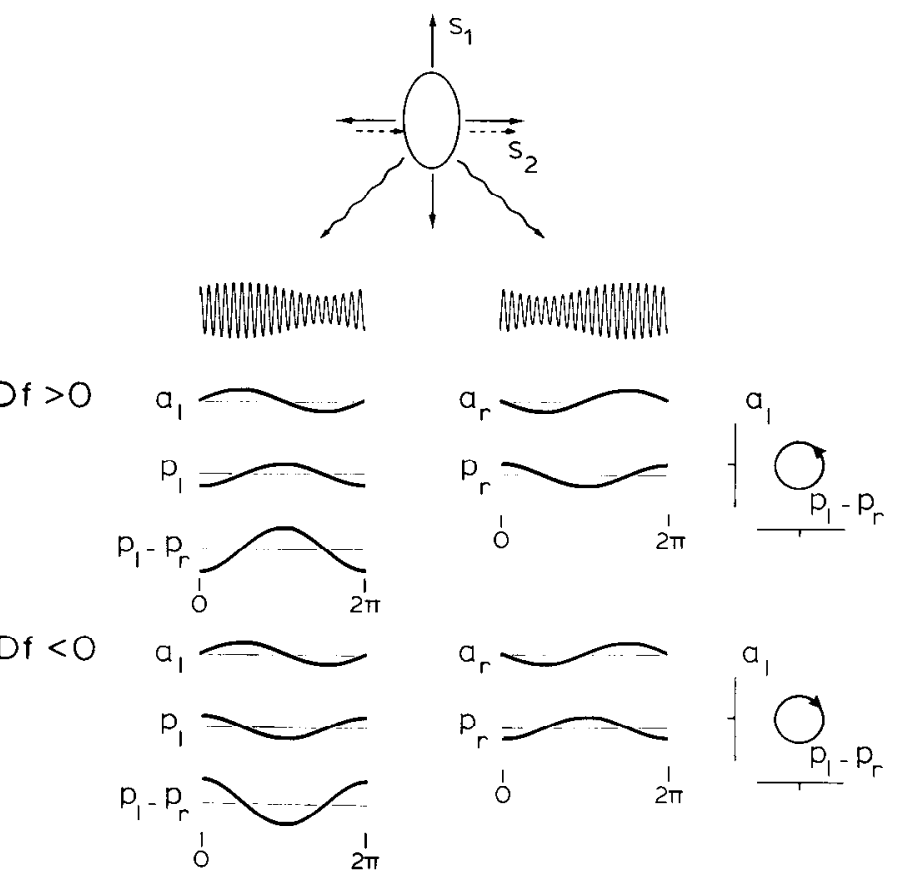

Figure 10. An external stimulus field, $\mathrm{S}_{2}$, yields maximal modulations in the phase difference $\left(p_{l}-p_{r}\right)$ between sides of the body which are opposite to each other with respect to the direction of $\mathrm{S}_{2}$. The diagram at the top indicates a cross-section through the fish's body which is exposed to a transverse $\mathbf{S}_{2}$ stimulus field (dashed vectors). The animal's own electric field $\left(\mathrm{S}_{1}\right)$ has an internal source, and its current thus crosses the skin with identical polarity in all parts of the body surface (solid vectors). In contrast, the $\mathrm{S}_{2}$ stimulus has opposite polarities on the left and right sides of the body. Consequently, when $S_{1}$ and $S_{2}$ are in phase on the right side of the body (maximizing the instantaneous stimulus amplitude), they are in antiphase on the left side of the body (minimizing stimulus amplitude). Since the frequency of $\mathrm{S}_{2}$ differs slightly from that of $S_{1}$, the opposite relation between current polarities will be reached half a beat cycle later. The beat pattern recorded on the left side of the body (schematically shown at the end of the undulating arrow), consisting of modulations in instantaneous amplitude $\left(a_{l}\right)$ and phase $\left(p_{l}\right)$ therefore is displaced in time by half a beat cycle with respect to the pattern simultaneously recorded on the right. The phase modulations, $p_{l}$ and $p_{r}$, are measured with reference to a site on the body surface without $S_{2}$ stimulation, such as the back or the belly. Phase modulations twice as large are obtained if the phase difference between opposite sides of the body is taken, $p_{l}-p_{r}$. With the frequency of $S_{2}$ being higher than that of $S_{1}$, i.e., with $D f>0$, a plot of $a_{l}$ versus $p_{l}$, or of $a_{r}$ versus $p_{r}$, yields a counterclockwise rotation. The same sense of rotation is obtained if $a_{1}$ is plotted versus $p_{l}-p_{r}$ (see circular graph at the right). Reversal of the sign of Df (bottom set of diagrams) only reverses the pattern of phase modulations, and any neuron which changes its firing pattern within the beat cycle in response to a sign reversal of $D f$ must therefore evaluate differential phase. Maximal $D f$-sign sensitivity should be obtained for an $\mathrm{S}_{2}$ orientation which yields maximal modulations in differential phase between the areas of body surface being compared by this neuron. Minimal $D f$-sign sensitivity should be obtained for an $\mathrm{S}_{2}$ orientation perpendicular to the former.

this set of experiments, $S_{1}$ and $S_{2}$ were not isolated from one another, pure phase modulations could no longer be generated. In order to increase the diversity of $S_{2}$ orientations, we added a vertical orientation by placing one electrode underneath the fish's head and one electrode above its tail section. In contrast to these forms of stimulation with "differential geometry," i.e., with $S_{1}$ and $S_{2}$ being applied through different sets of electrodes and thus having different field geometries, we could also stimulate with "identical geometry" by adding $S_{1}$ and $S_{2}$ through the same pair of electrodes. With this last form of stimulation the intensity ratio between $S_{1}$ and $S_{2}$ is identical everywhere.
Modulations of differential phase are therefore absent, and amplitude is modulated alone. Thus, under this stimulus regimen, we could test for pure amplitude modulations $\left(S_{1}\right.$ and $S_{2}$ delivered through the same pair of electrodes) or the natural situation of combined modulations of differential phase and amplitude $\left(S_{1}\right.$ and $S_{2}$ delivered through distinct pairs of electrodes).

The experiments by Partridge et al. (1981) had demonstrated that many cells in the deeper laminae of the torus discriminate the sign of $D f$ (the difference in frequency between the animal's own signal and a foreign signal, $S_{2}$ ) most clearly if the $S_{2}$ field is oriented in a particular direction with respect to the animal's body axis. An orientation perpendicular to this direction yielded almost no discrimination. This result suggested that these cells compared stimulus phase from particular parts of the body surface whose phase difference was maximally modulated under a particular orientation of the $\mathrm{S}_{2}$ field (see Fig. 10). We applied this stimulus paradigm again in our intracellular study in order to identify cell types which are only sensitive to amplitude modulations and therefore do not discriminate the sign of $D f$, regardless of the orientation of the $S_{2}$ stimulus field. An additional purpose of this new series of experiments was to anatomically identify those cell types which are sensitive to amplitude and phase and therefore discriminate the sign of $D f$ for appropriate orientations of the $\mathrm{S}_{2}$ stimulus field.

The following results were obtained. First, we identified cell types which, at any given orientation of the $S_{2}$ field, responded with identical firing patterns for positive and negative $D f \mathrm{~s}$. Depending upon their responses to amplitude modulations, these cells could be classified as either E- or I-type. Also, much as $E$ - and I-afferents from the ELLL, these cells responded most strongly for one particular orientation of the $S_{2}$ field, obviously a consequence of the location of their receptive field on the body surface. (Note that a transverse $S_{2}$ field yields maximal beat modulations on the sides of the body, whereas a longitudinal $\mathrm{S}_{2}$ field yields maximal modulations on the face.) The responses of these cells under the most effective $S_{2}$ orientation were predictable from their responses to stimulation with identical stimulus geometry, with $S_{1}$ and $S_{2}$ presented between an internal and an external electrode (so that all parts of the body experienced a sufficient modulation in stimulus amplitude and no modulations in differential phase). These cells were thereby shown to be driven by amplitude modulations alone. Most importantly, two of these cells types (Fig. 11) project to the tectum. This means that the tectum also receives, in addition to information about differential phase provided by the cell types shown in Figures 7 to 9, pure and somatotopically ordered information about modulations of stimulus amplitude.

Second, many cells responded differentially for positive and negative $D f \mathrm{~s}$, and, much as was observed in earlier extracellular studies (Partridge et al., 1981), the degree of $D f$-sign sensitivity depended upon the orientation of the $\mathrm{S}_{2}$ field. Under stimulation with identical gcometry, i.e., in the absence of differential phase modulations, these cells, if they responded at all, could be classified as E- or I-types. These cells were either local interneurons or larger cells which projected to the tectum and anatomically resembled those shown in Figure 9.

A summary of toral cell types which encode information essential for the JAR is shown in Figure 12. The tectum receives pure, somatotopic amplitude information via cells in lamina 5 and cells in the vertical neuropil of lamina 6 (see Fig. 11). It receives almost pure differential phase information via a cell type in lamina $8 b$ and $8 c$ (see Fig. 7), as well as combined information about amplitude and phase via additional cell types in lamina $8 \mathrm{~b}$ and $8 \mathrm{c}$ (see Figs. 8 and 9). This information is sufficient for the control of the JAR (see heavily framed responses in Fig. 12). 


\section{Discussion}

Neuronal substrates of the JAR. The JAR is one of several tasks accomplished by the torus, and many neurons which most likely participate in the control of the JAR may also be part of networks serving other functions, such as the detection of moving objects. Although we have no direct proof that a given class of neurons participates in the JAR (stimulation of single cells has no behavioral effect and stimulation of larger subsets is impossible), we nevertheless have strong indirect evidence for their role. The cells described in this study specifically and vigorously respond to stimulus features which, on the basis of behavioral experiments, have been found to be crucial for the JAR (Heiligenberg et al., 1978; Heiligenberg, 1980; Heiligenberg and Bastian, 1980).

It is our goal to ultimately identify all major elements in the neuronal loop responsible for the JAR. Since this behavior persists in a neurophysiological preparation, the objective of understanding the processing of relevant sensory information and the generation of the motor output seems feasible. In the present study, we identified toral neurons which are phase sensitive. That is, these units fire preferentially for particular timing relations between sinusoidal electric signals experienced in different parts of the sensory surface. Combining such phase computations with the processing of concurrent amplitude modulations provides the necessary information to determine the sign and the magnitude of $D f$ (see Fig. 12). Yet, as was expected on the basis of previous studies (Partridge et al., 1981), $D f$-sign-selective neurons (i.e., neurons which discharge preferentially for one particular sign of $D f$ ) either do not exist in the torus or are extremely rare. Scheich (1974) reported a $D f$-sign-selective neuron from the torus of Eigenmannia. However, since he did not identify his recording sites histologically, it is possible that he actually recorded from fibers in the vicinity of the torus. Thus, we still have no strong evidence that the sign of $D f$ is determined at the level of the torus.

From the anatomical reconstruction of physiologically identified cells it is clear that information about modulations of local stimulus amplitude and differential phase is encoded by several types of toral neurons which project to the optic tectum (see Figs. 9, 11, and 12). The tectum, which is known to integrate electric images into a multimodal map of stimulus space (Bastian, 1982), is also involved in the control of the JAR. Ablation of the tectum, without damage to the underlying torus, instantly and permanently abolishes the JAR (unpublished observation). Moreover, we have identified tectal cells which fire only for one sign of $D f$ and only discriminate the sign of $D f$ in this manner under stimulus regimens which yield JARs; i.e., these units do not, for example, respond if the two interfering stimuli have identical stimulus field geometries and thus do not provide modulations in differential phase (Rose and Heiligenberg, 1984). These $D f$-sign-specific tectal cells appear to project to the nucleus electrosensorius complex which contains cells with similar properties and which, in turn, projects to the prepacemaker nucleus of the midbrain (Bastian and Yuthas, 1984). The prepacemaker nucleus, which controls the pacemaker nucleus in the medulla (Heiligenberg et al., 1981), thus links the chain of neuronal elements underlying sensory processing and motor control of the IAR.

General design considerations. What is surprising is the seemingly unnecessary complexity of the neuronal machinery involved in a task as simple as the JAR. We have found no trace of a neuronal circuit as simple as those proposed earlier (Bastian and Heiligenberg 1980a, b; Heiligenberg and Bastian, 1980; Partridge et al., 1981) and instead are facing a multitude of laminae and classes of neurons which gradually, through many stages, extract from the flow of electrosensory information features which are necessary for the JAR. The reason for this complexity may lie in the evolution of this system. We assume that the JAR has evolved from more general motor responses to moving electric images. The ohmic load of an object which differs electrically from the surrounding water modulates the local amplitude of the animal's EOD while the capacitive load of the object introduces local phase shifts. Such phase shifts can best be detected by comparison of the timing of zerocrossings in different parts of the body surface, i.e., by the evaluation of differential phase (Scheich and Bullock, 1974). The abilities to monitor modulations in local amplitude and differential phase thus appear to be early components in the evolution of the electrolocation system which could readily be adapted for the assembly of additional hardware for the JAR. The neuronal machinery of the JAR therefore may have emerged by modifications of and additions to an existing machinery already adapted to functions of electrolocation, and this may be the reason that it is not engineered in the most straightforward manner.

Earlier extracellular studies on toral cells by Bastian and Heiligenberg (1980a, b) and Partridge et al. (1981) indicated that toral neurons lack the temporal precision, sensitivity, and sensory specificity expressed in the JAR. These properties of the JAR appear to emerge statistically from large populations of less sensitive and less specifically tuned neurons in the torus. It is only further along the sensory input to motor output pathway, at the level of the nucleus electrosensorius, that neurons are found which more closely reflect dynamic properties and sensory specificities of the JAR (Bastian and Yuthas, 1984).

Analogies to the auditory system. The clear separation of neuronal systems for the processing of phase and amplitude information resembles a similar separation in the auditory system of the owl (Moiseff and Konishi, 1981, 1983; Sullivan and Konishi, 1984; Takahashi et al., 1984; M. Konishi, W. E. Sullivan, and T. Takahashi, manuscript in preparation). In both instances, separate networks relay these two forms of information from the hindbrain to analogous structures in the midbrain, the dorsal torus in gymnotoid fish and the inferior colliculus in birds, where phase and amplitude are processed jointly for more complex tasks, the JAR in the fish and directional hearing in azimuth and elevation in the owl. In both instances, temporal disparities are evaluated with accuracies in the microsecond range. These are temporal disparities between inputs from the two ears in the owl and between pairs of possibly hundreds of receptive fields on the body surface of the fish. Whereas electrical synapses account for high temporal acuity in the neuronal code of the fish, only chemical synapses apparently are involved in the bird (Jhaveri and Morest, 1982), and it is remarkable that such a system of even less accurately coding neurons can achieve such precise temporal resolution at the behavioral level.

Neurons in the torus are remarkably sensitive to modulations of stimulus amplitude. Even small modulations, on the order of $1 \%$, produce dramatic modulations in spike rate. This phenomenon is also observed in the processing of amplitudemodulated auditory signals in mammals and amphibians (Møller, 1972, 1973, 1974, 1977; Rose and Capranica, 1984). Furthermore, neurons in the ELLL and torus of Eigenmannia show selectivity to the rate of amplitude modulations, often taking the form of tuning to a particular rate of amplitude modulations (Bastian, 1981b; Partridge et al., 1981), much as do auditory neurons in the midbrain of amphibians (Rose and Capranica, 1983).

Problems of relating function and morpholngy. We are still far from understanding the wiring of the torus in its entirety. In current Golgi studies by C. E. Carr and L. Maler (submitted for publication), 50 cell types have been identified, distributed over the 12 laminae of the dorsal torus (laminae 1 to 9 , with 
(a)

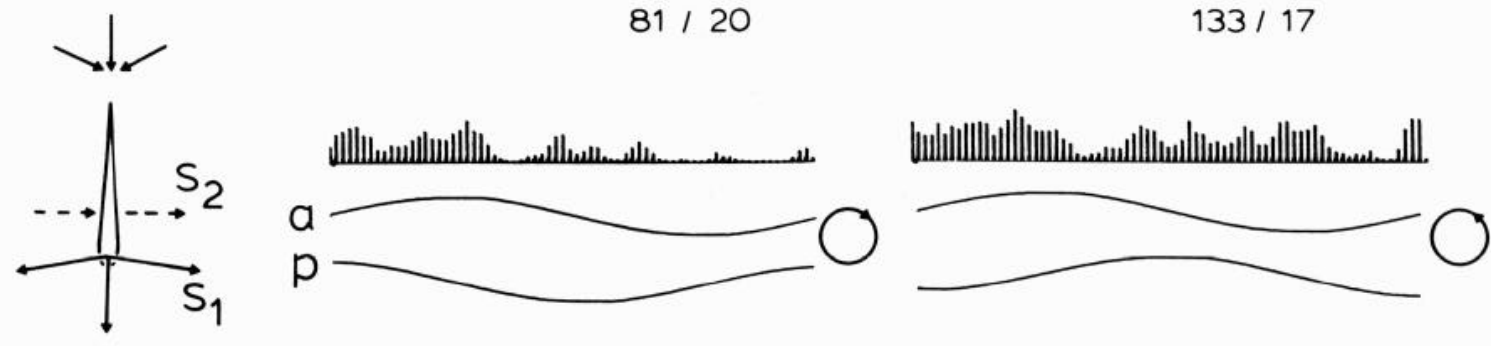

(b)

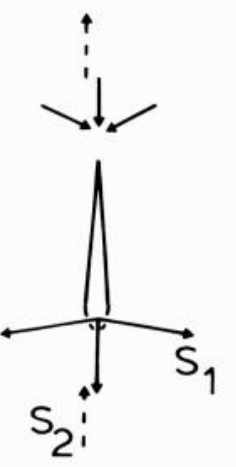

$38 / 7$

$65 / 10$
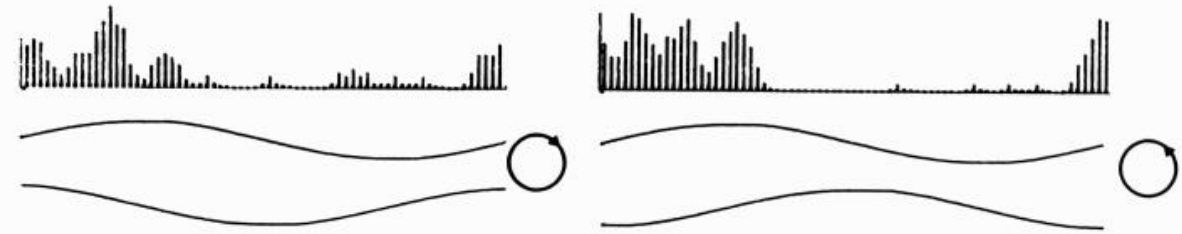

(C)

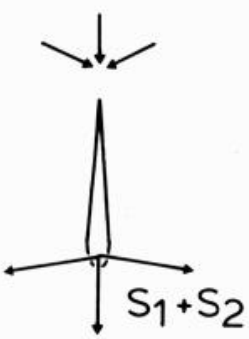

$121 / 19$

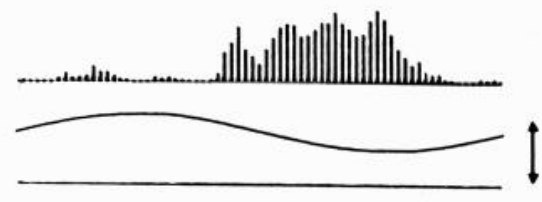

(d)

(e)
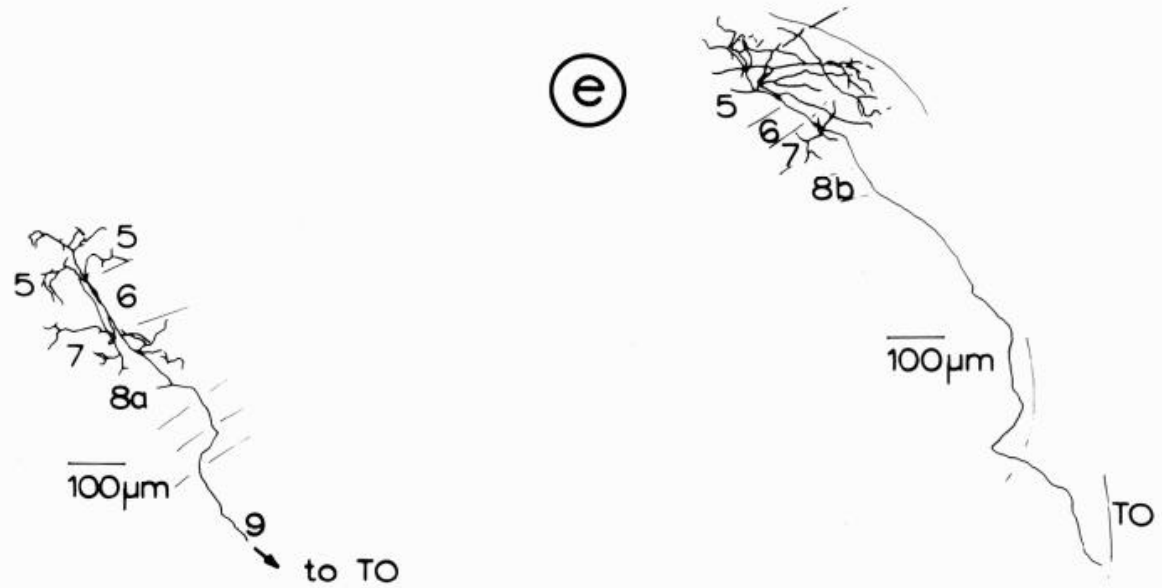

Figure 11 


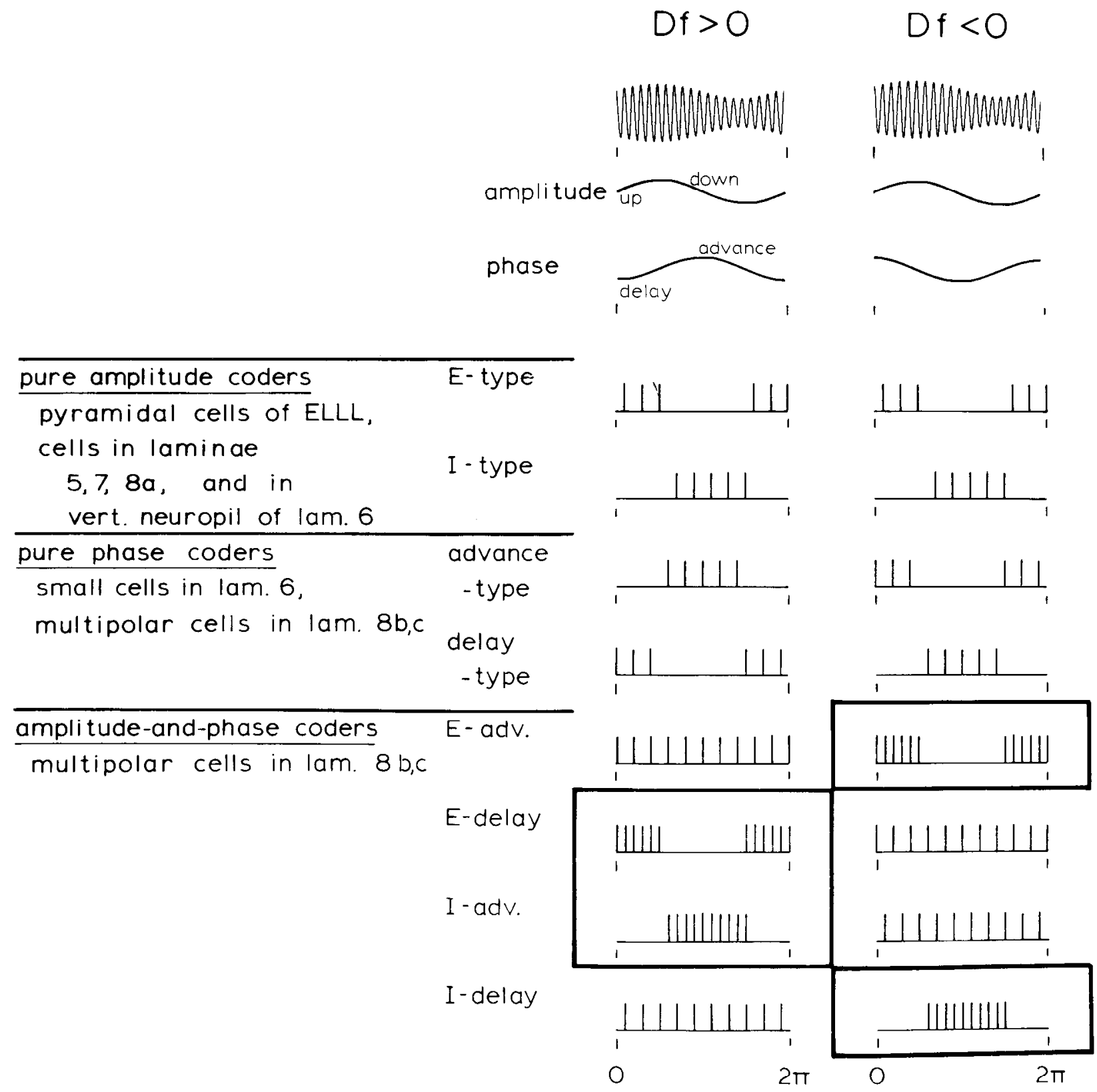

Figure 12. The classification of toral cells as coders of amplitude and phase information contained in beat patterns (top) for positive and negative $D f$. E-delay and I-advance cells reach highest spike densities for positive $D f s$, whereas E-advance and I-delay cells reach highest spike densities for negative $D f s$ (patterns framed by thick lines). Pure amplitude coders in lamina 5 and in the vertical neuropil of lamina 6 as well as phase and amplitude- and-phase coders in laminae $8 \mathrm{~b}$ and $8 \mathrm{c}$ project to the optic tectum.

lamina 8 consisting of 8 a through $8 \mathrm{~d}$ ). A seemingly simple task, the study of neuronal connections in lamina 6 underlying the computation of differential phase has proven exceedingly difficult due to the "small" cells (see Fig. 4), which are crucial for this computation (Carr et al., 1983). Although our studies have demonstrated that the morphologically distinct cell types of this lamina, the spherical cell afferents, giant cells, and small cells, correspond to functionally distinct classes, the relation between structure and function for the cells of the remaining laminae is not clear. Although we can characterize laminae

Figure 11. Responses of a cell in the vertical neuropil of lamina 6. This cell is insensitive to modulations in phase and responds to modulations in amplitude in the manner of an I-type unit. Histograms of spike rates under a transverse $\mathrm{S}_{2}$ stimulus field (dashed vectors in inset at left) (a), under a longitudinal $\mathrm{S}_{2}$ field $(b)$, and under identical stimulus geometry $(c)$ are shown in the manner of Figure 5 . The beat rate was 2 Hz. The peak of the amplitude characteristic (first line below histograms) corresponds to the moment where the $S_{1}$ and $S_{2}$ stimulus generators are in phase. At this point, the stimulus amplitude is maximal under differential stimulus geometry $\left(a\right.$ and $b$ ) only in parts of the field where the $S_{1}$ and $S_{2}$ vectors form acute angles. The amplitude is minimal where this angle is obtuse. The experiment in $c$ shows that this cell is of the I-type (it fires during the falling phase of the amplitude). That this cell fires in $b$ during the rising phase of the amplitude indicates that its receptive field is located in the region of the face, where $S_{1}$ and $S_{2}$ form an obtuse angle. The cell fires with nearly identical patterns for both senses of rotation under $b$ and hardly locks into the beat cycle under $a$. $d$ shows a camera lucida drawing of a cell in the vertical neuropil of lamina 6 . $e$ shows a cell of lamina 5 which responds in a similar manner under these stimulus regimens. Numbers indicate laminae of the torus. TO, is the optic tectum. 
with regard to the predominant functional properties of their cells, such as coding of amplitude in laminae 5 and 7 and coding of phase and amplitude in lamina $8, \mathrm{~b}$ and $\mathrm{c}$, a strict correlation between cell morphology and function is still beyond our grasp.

Excluding the giant cells of lamina 6 and afferents to the torus, we have intracellularly labeled and physiologically identified well over 120 toral neurons which fall into approximately 30 types. Most of these cell types, which will be described in detail in a subsequent publication, are local interneurons that either lie within a single lamina or connect different laminae, apparently relaying information about amplitude and phase between different parts of the somatotopic map of the body surface or between different laminar processing levels of the torus. Since we deliberately labeled only those cells which responded to our limited set of stimulus regimens, we excluded cell types which probably would have responded to stimulus regimens, such as moving objects, that control other behaviors. In view of the 30 or more types of cells which we have found responding to JAR-related stimuli, it is apparent that a much larger sample of labeled cells is required in order to recognize these cell types reliably. In the case of the multipolar cells of lamina 8, b and c (see Fig. 9), we find that cells with similar morphology are physiologically distinct. It is conceivable that a larger sample size will allow us to identify some subtle differences in their morphology that have so far escaped us (Katz, 1984). It is also possible that these cells are morphologically identical and that differences in their physiology are accidental to statistical rules in the establishment of their connections during development.

\section{References}

Bastian, J. (1981a) Electrolocation. I. How the electroreceptors of Apteronotus albifrons code for moving objects and other electrical stimuli. J. Comp. Physiol. 144: 465-479.

Bastian, J. (1981b) Electrolocation. II. The effects of moving objects and other electrical stimuli on the activities of two categories of posterior lateral line lobe cells in Apteronotus albifrons. J. Comp. Physiol. 144: 481-494.

Bastian, J. (1982) Vision and electroreception: Integration of sensory information in the optic tectum of the weakly electric fish Apteronotus albifrons. J. Comp. Physiol. 147: 287-297.

Bastian, J., and W. Heiligenberg (1980a) Neural correlates of the jamming avoidance response in Eigenmannia. J. Comp. Physiol. 136 135-152.

Bastian, J., and W. Heiligenberg (1980b) Phase sensitive midbrain neurons in Eigenmannia: Neural correlates of the jamming avoidance response. Science 209: 828-831.

Bastian, J., and J. Yuthas (1984) The jamming avoidance response of Eigenmannia: Properties of a diencephalic link between sensory processing and motor output. J. Comp. Physiol. 154: 895-908.

Bennett, M. L. V. (1971) Electric organs. In Fish Physiology, W. S. Hoar and D. H. Randall, eds., Vol V, pp. 347-491, Academic Press, Inc., New York.

Bullock, T. H., and R. G. Northcutt (1982) A new electroreceptive teleost: Xenomystus nigri (Osteoglossiformes: Notopteridae). J. Comp. Physiol. 148: 345-352.

Bullock, T. H., R. H. Hamstra, and H. Scheich (1972) The jamming avoidance response of high frequency electric fish. J. Comp. Physiol. 77: $1-48$.

Bullock, T. H., K. Behrend, and W. Heiligenberg (1975) Comparison of the jamming avoidance responses in gymnotoid and gymnarchid electric fish: A case of convergent evolution of behavior and its sensory basis. J. Comp. Physiol. 103: 97-121.

Carr, C. E., L. Maler, W. Heiligenberg, and E. Sas (1981) Laminar organization of the afferent and efferent systems of the torus semicircularis of gymnotiform fish: Morphological substrates for parallel processing in the electrosensory system. J. Comp. Neurol. 203: 649670.

Carr, C. E., L. Maler, and E. Sas (1982) Peripheral organization and central projections of the electrosensory nerves in gymnotiform fish. J. Comp. Neurol. 211: 139-153.

Carr, C. E., B. Taylor, and L. Maler (1983) Ultrastructure of a phase comparison circuit in the midbrain of the weakly electric fish Eigenmannia. Soc. Neurosci. Abstr. 9: 1028.

Heiligenberg, W. (1980) The jamming avoidance response in the weakly electric fish Eigenmannia. Naturwissenschaften 67: 499-507.

Heiligenberg, W. (1983) The jamming avoidance response in an electric fish: Algorithms in sensory information processing and their neuronal organization. In Advances in Vertebrate Neuroethology, J. -P. Ewert, D. Ingel, and R. Capranica, eds., pp. 669-699, Plenum Press, New York.

Heiligenberg, W., and J. Bastian (1980) The control of Eigenmannia's pacemaker by distributed evaluation of electroreceptive afferences. J. Comp. Physiol. 136: 113-133.

Heiligenberg, W., and J. Bastian (1984) The electric sense of weakly electric fish. Annu. Rev. Physiol. 46: 561-583.

Heiligenberg, W., and J. Dye (1982) Labelling of electroreceptive afferents in a gymnotoid fish by intracellular injection of HRP: The mystery of multiple maps. J. Comp. Physiol. 148: 287-296.

Heiligenberg, W., C. Baker, and J. Matsubara (1978) The jamming avoidance response in Eigenmannia revisited: The structure of a neuronal democracy. J. Comp. Physiol. 127: 267-286.

Heiligenberg, W., 'T. Finger, J. Matsubara, and C. E. Carr (1981) Input to the medullary pacemaker nucleus in the weakly electric fish, Eigenmannia (Sternopygidae, Gymnotiformes). Brain Res. 211: 418423.

Jhaveri, S. R., and D. K. Morest (1982) Neuronal architecture in the nucleus magnocellularis of the chicken auditory system with observations on nucleus laminaris: A light and electron microscope study. Neuroscience 7: 809-835.

Katz, L. C. (1984) Intrinsic connectivity of identified projection neurons in cat visual cortex brain slices. Ph.D. thesis, California Institute of Technology, Pasadena, CA.

Maler, L., E. Sas, and J. Rogers (1981) The cytology of the posterior lateral line lobe of high frequency weakly electric fish (Gymnotoidei): Dendritic differentiation and synaptic specificity in a simple cortex. J. Comp. Neurol. 195: 87-140.

Maler, L., E. Sas, E. C. Carr, and J. Matsubara (1982) Efferent projections of the posterior lateral line lobe in gymnotiform fish. I. Comp. Neurol. 211: 154-164.

Moiseff, A., and M. Konishi (1981) Neuronal and behavioral sensitivity to binaural time differences in the owl. J. Neurosci. 1: 4048.

Moiseff, A., and M. Konishi (1983) Binaural characteristics of units in the owl's brainstem auditory pathway: Precursors of restricted spatial receptive fields. J. Neurosci. 3: 2553-2562.

Møller, A. R. (1972) Coding of amplitude- and frequency-modulated tones in the cochlear nucleus of the rat. Acta Physiol. Scand. 86. $223-238$.

Møller, A. R. (1973) Statistical evaluation of the dynamic properties of cochlear nucleus units using stimuli modulated with pseudorandom noise. Brain Res. 57: 443-456.

Møller, A. R. (1974) Responses of units in the cochlear nucleus to sinusoidally amplitude-modulated tones. Exp. Neurol. 45: 104-117.

Møller, A. R. (1977) Coding of time-varying sounds in the cochlear nucleus. Audiology 17: 446-468.

Partridge, B. L., W. Heiligenberg, and J. Matsubara (1981) The neural basis of a sensory filter in the jamming avoidance response: No Grandmother Cells in sight. J. Comp. Physiol. 145: 153-168.

Rose, G., and R. R. Capranica (1983) Temporal selectivity in the central auditory system of the leopard frog. Science 219: 1087-1089.

Rose, G., and R. R. Capranica (1984) Processing amplitude-modulated sounds by the auditory midbrain of two species of toads: Matched temporal filters. J. Comp. Physiol. 154: 211-219.

Rose, G., and W. Heiligenberg (1984) Neural correlates of the jamming avoidance response: Df-sign-specific neurons in the optic tectum of the electric fish Eigenmannia. Soc. Neurosciences Abstr. 10: 403.

Saunders, J., and J. Bastian (1984) The physiology and morphology of two types of electrosensory neurons in the weakly electric fish Apteronotus leptorhynchus. J. Comp. Physiol. 154: 197-209.

Scheich, H. (1974) Neural analysis of wave form in the time domain: Midbrain units in electric fish during social behavior. Science 185 : 365-367.

Scheich, H., and T. H. Bullock (1974) The role of electroreceptors in the animal's life. II. The detection of electric fields from electric organs. In Handbook of Sensory Physiology, A. Fessard, eds., Vol. III, Sect. 3, pp. 201-256, Springer-Verlag, Berlin.

Scheich, H., and S. O. E. Ebbesson (1981) Inputs to the torus semicir- 
cularis in the electric fish Eigenmannia virescens. Cell 'Tissue Res. 215: 531-536.

Scheich, H., and S. O. E. Ebbesson (1984) The multimodal torus in the weakly electric fish, Eigenmannia. In Advances in Anatomy, Embryology and Cell Biology, F. Beck, W. Hild, J. von Lindborgh, R. Ortmann, D. E. Pauly, and T. H. Schiebler, eds., Vol. 82, pp. 1-69, Springer-Verlag, Berlin.

Scheich, H., T. H. Bullock, and R. H. Hamstra (1973) Coding properties of two classes of afferent nerve tibers: High frequency electroreceptors in the electric fish, Eigenmannia. J. Neurophysiol. 36: 39-60.

Sullivan, W. E., and M. Konishi (1984) Segregation of stimulus phase and intensity coding in the cochlear nucleus of the barn owl. $J$. Neurosci. 4: 1787-1799.

Takahashi, T., A. Moiseff, and M. Konishi (1984) Time and intensity cues are processed independently in the auditory system of the owl. J. Neurosci. 4: 1781-1786. 